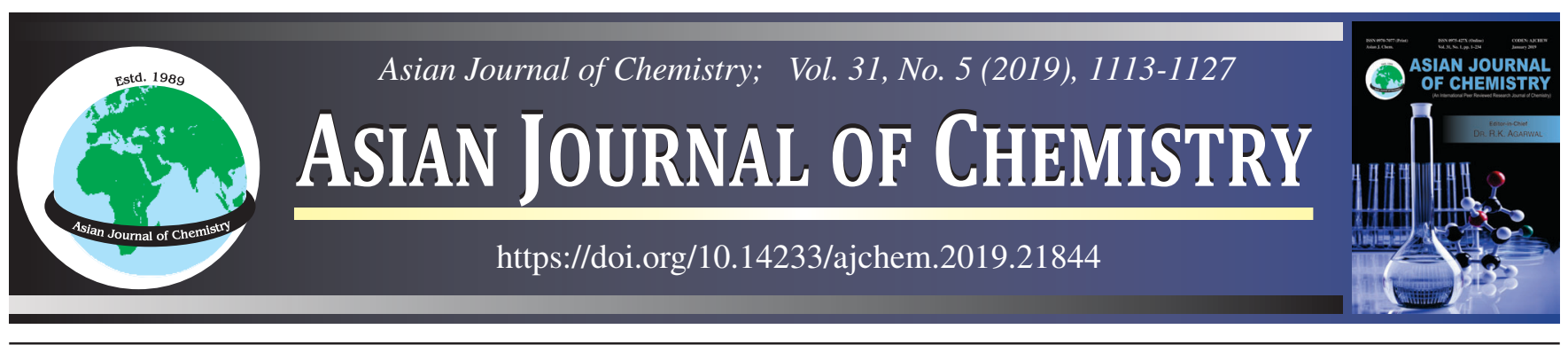

\title{
Studies on Acoustic and Aggregation Properties of Sodium Dodecyl Sulfate in Amino Acid Solutions through Ultrasonic Velocity Technique
}

\author{
M. Roksana Khatun ${ }^{1, *, \bullet}$, M. Monirul Islam ${ }^{2}$, Md. Nazrul Islam ${ }^{2}$, Md. Masudur Rhaman $^{1}$ and Ranjit K. Nath ${ }^{1, *, \bullet}$
}

${ }^{1}$ Department of Chemistry, Faculty of Engineering \& Technology, Chittagong University of Engineering \& Technology, Chattogram-4349, Bangladesh ${ }^{2}$ Department of Chemistry, Faculty of Science, University of Rajshahi, Rajshahi-6205, Bangladesh

*Corresponding authors: E-mail: ark19mrk@gmail.com; rkn_chem@yahoo.com

Received: 27 November 2018; Accepted: 17 January 2019;

Published online: 28 March 2019;

AJC-19341

Acoustic and aggregation properties of sodium dodecyl sulfate in aqueous amino acid systems have been studied through ultrasonic velocity techniques at different temperature as a function of concentration and at different temperature $(288.15,293.15,298.15,303.15$, $308.15,313.15,318.15$ and $323.15 \mathrm{~K}$ ) and atmospheric pressure using density and sound velocity analyzer (DSA 5000, Anton paar, Austria). Densities and ultrasonic velocity of sodium dodecyl sulfate in aqueous amino acid have been measured and from the experimental data, adiabatic compressibility $\left(\beta_{\mathrm{s}}\right)$, apparent molar adiabatic compressibility $\left(\phi_{\mathrm{k}}\right)$, critical micelle concentration $(\mathrm{CMC})$, acoustic impedance $(Z)$, relative association $\left(R_{A}\right)$ and molar sound velocity $\left(R_{m}\right)$ have been calculated. The results show significant information about acoustic properties of sodium dodecyl sulfate (solute-solute interactions, aggregation behaviour, relative association and structure formation) in aqueous and aqueous amino acid solution.

Keywords: Sodium dodecyl sulfate, Glycine, DL-alanine, Apparent molar volume, Critical micelle concentration, Hydration number. ᄂ

\section{INTRODUCTION}

Surfactant, also called surface-active agent, that when added to a liquid, reduces its surface tension, thereby increasing its spreading and wetting properties. Surfactants have vast application in pharmaceuticals, biotechnology, agriculture, food industry and cosmetics industry [1-3]. But their interaction with various types of co-solutes, biological macromolecules is not clearly understood. So, it is important to investigate the interaction of sodium dodecyl sulfate (SDS) with various types of molecules in applied medium. Surfactants interact with working medium molecules through their head group and hydrophobic tail. Micellar properties of surfactant make more complexities in working medium but very operative toward many applications [4-8].

The studies of surfactant with amino acids have important fundamental and useful research interest [9-11]. The interaction of surfactant with protein has been investigated using various methods [12-14]. Surfactant interacts with protein and bind them leading to substantial changes in protein conformation.
Surfactant modifies the protein structure and changes the function or character of surfactant-protein interaction. Several phenomena on the interaction mode of surfactants with proteins until unanswered. So, it is definitely important to understand the nature of surfactant-protein interactions.

Sodium dodecyl sulfate has a special place in laboratory and industrial use. Surfactant has unique character because of their hydrophilic and hydrophobic groups in the same molecule. It can bring conformational changes and modify the activities of many proteins [15-20]. To the best of our knowledge, no report has been available previously on the acoustic properties of SDS in aqueous amino acid solutions. Surfactant has unique character because of their hydrophilic and hydrophobic groups in the same molecule. In this paper, we explain the acoustic and aggregation properties of SDS in the various concentrations of glycine and DL-alanine through ultrasonic technique [21-24]. Among the various properties of physical parameter, acoustic $[25,26]$ and aggregation property can give us potential information about structural changes of SDS in amino acid solutions.

This is an open access journal, and articles are distributed under the terms of the Creative Commons Attribution-NonCommercial-ShareAlike 4.0 (CC BY-NC-SA 4.0) International License which allows readers to freely read, download, copy, distribute, print, search, or link to the full texts of its articles and to use them for any other lawful non-commercial purpose as long as the original source is duly acknowledged. 


\section{EXPERIMENTAL}

Sodium dodecyl sulfate (purity, mass fraction $>0.99$ ), glycine (purity, mass fraction $>0.99$ ) and DL-alanine (purity, mass fraction > 0.99) obtained from Fluka Chemical Company, Switzerland were used in this study. The specifications of the chemicals used in this study are given in Table- 1 .

In the previous literature the $\mathrm{CMC}$ value of aqueous SDS was reported around $0.009 \mathrm{~m}$ at $25^{\circ} \mathrm{C}$ and mean aggregation number vary from 58 to 64 . Distilled water (supplied) was purified by redistilled and deionized process using two ion exchange columns. The deionized water was further distilled in alkaline $\mathrm{KMnO}_{4}$ medium. Conductivity of this purified water was $5 \times$ $10^{-6} \mathrm{~S} \mathrm{~cm}^{-1}$. For weighing, electric balance with an accuracy of $\pm 0.0001 \mathrm{~g}$ was used. For measurement of density and ultrasonic velocity, a densitometer (DSA-5000, Anton Paar, Austria) was used. Each solution was prepared by weight immediately before the measurement.

\section{RESULTS AND DISCUSSION}

The densities $(\rho)$ and ultrasonic velocity (u) of SDS in aqueous and aqueous amino acid solutions are measured at different concentration of solutes and temperature, also presented in Tables 2 and 3.

Adiabatic compressibility $\left(\beta_{\mathrm{S}}\right)$ and apparent molar adiabatic compressibility $\left(\phi_{\mathrm{K}}\right)$ : Adiabatic compressibility $\left(\beta_{\mathrm{s}}\right)$, was calculated by densities and ultrasonic velocity data using eqn. 1:

TABLE-1

SPECIFICATIONS OF THE CHEMICALS

\begin{tabular}{lccc}
\hline \multicolumn{1}{c}{ Chemical name } & Molar mass $\left(\mathrm{kg} \mathrm{mol}^{-1}\right)$ & Purity declared by manufacturer & Origin \\
\hline Sodium dodecyl sulfate & 0.28838 & Mole fraction $\geq 0.99$ & Fluka Chemical Company, Switzerland \\
Glycine & 0.07507 & Mole fraction $\geq 0.99$ & Fluka Chemical Company, Switzerland \\
DL-alanine & 0.14614 & Mole fraction $\geq 0.99$ & Fluka Chemical Company, Switzerland \\
\hline
\end{tabular}

TABLE-2

DENSITIES $\left(\rho, \mathrm{kg} \mathrm{m}^{-3}\right)$ OF SDS IN AQUEOUS AND AQUEOUS AMINO ACID SOLUTIONS AT $288.15,293.15,298.15,303.15,308.15$ AND 313.15 K, RESPECTIVELY

\begin{tabular}{|c|c|c|c|c|c|c|}
\hline $\mathrm{m}\left(\mathrm{mol} \mathrm{kg}{ }^{-1}\right)$ & $298.15 \mathrm{~K}$ & $303.15 \mathrm{~K}$ & $308.15 \mathrm{~K}$ & $313.15 \mathrm{~K}$ & $318.15 \mathrm{~K}$ & $323.15 \mathrm{~K}$ \\
\hline \multicolumn{7}{|c|}{ Water + SDS } \\
\hline 0.00000 & 999.101 & 998.206 & 997.045 & 995.646 & 994.032 & 992.215 \\
\hline 0.00034 & 999.138 & 998.239 & 997.080 & 995.679 & 994.063 & 992.246 \\
\hline 0.00089 & 999.171 & 998.272 & 997.109 & 995.708 & 994.094 & 992.273 \\
\hline 0.00136 & 999.189 & 998.289 & 997.124 & 995.724 & 994.108 & 992.288 \\
\hline 0.01499 & 999.932 & 999.007 & 997.823 & 996.400 & 994.766 & 992.932 \\
\hline 0.02999 & 1000.638 & 999.694 & 998.491 & 997.054 & 995.406 & 993.558 \\
\hline 0.04398 & 1001.294 & 1000.330 & 999.111 & 997.660 & 995.999 & 994.138 \\
\hline 0.05807 & 1001.942 & 1000.959 & 999.725 & 998.258 & 996.580 & 994.706 \\
\hline 0.07199 & 1002.582 & 1001.580 & 1000.327 & 998.845 & 997.155 & 995.273 \\
\hline 0.08594 & 1003.218 & 1002.201 & 1000.932 & 999.436 & 997.731 & 995.835 \\
\hline 0.10085 & 1003.897 & 1002.860 & 1001.571 & 1000.062 & 998.345 & 996.433 \\
\hline 0.11498 & 1004.497 & 1003.443 & 1002.140 & 1000.615 & 998.886 & 996.965 \\
\hline 0.12860 & 1005.091 & 1004.022 & 1002.706 & 1001.166 & 999.424 & 997.491 \\
\hline 0.14257 & 1005.672 & 1004.584 & 1003.252 & 1001.702 & 999.945 & 998.002 \\
\hline 0.15700 & 1006.295 & 1005.189 & 1003.841 & 1002.276 & 1000.507 & 998.549 \\
\hline 0.17194 & 1007.184 & 1005.953 & 1004.580 & 1002.988 & 1001.199 & 999.222 \\
\hline 0.18610 & 1007.654 & 1006.463 & 1005.084 & 1003.485 & 1001.687 & 999.707 \\
\hline 0.19845 & 1008.073 & 1006.918 & 1005.524 & 1003.916 & 1002.109 & 1000.121 \\
\hline \multicolumn{7}{|c|}{ Water + Glycine $(0.049 \mathrm{~m})+$ SDS } \\
\hline 0.00000 & 1000.719 & 999.801 & 998.623 & 997.209 & 995.582 & 993.754 \\
\hline 0.00035 & 1000.760 & 999.842 & 998.662 & 997.247 & 995.619 & 993.793 \\
\hline 0.00062 & 1000.780 & 999.861 & 998.679 & 997.266 & 995.635 & 993.806 \\
\hline 0.01394 & 1001.528 & 1000.584 & 999.382 & 997.951 & 996.306 & 994.464 \\
\hline 0.02828 & 1002.155 & 1001.194 & 999.980 & 998.531 & 996.874 & 995.020 \\
\hline 0.04222 & 1002.838 & 1001.862 & 1000.630 & 999.165 & 997.496 & 995.630 \\
\hline 0.05679 & 1003.466 & 1002.470 & 1001.222 & 999.748 & 998.063 & 996.186 \\
\hline 0.07115 & 1004.166 & 1003.152 & 1001.887 & 1000.394 & 998.698 & 996.807 \\
\hline 0.08490 & 1004.793 & 1003.766 & 1002.484 & 1000.977 & 999.266 & 997.365 \\
\hline 0.09953 & 1005.425 & 1004.376 & 1003.078 & 1001.559 & 999.837 & 997.921 \\
\hline 0.11068 & 1005.891 & 1004.826 & 1003.515 & 1001.982 & 1000.247 & 998.320 \\
\hline 0.12577 & 1006.537 & 1005.453 & 1004.147 & 1002.578 & 1000.830 & 998.891 \\
\hline 0.14084 & 1007.217 & 1006.117 & 1004.771 & 1003.210 & 1001.445 & 999.493 \\
\hline 0.14951 & 1007.620 & 1006.509 & 1005.153 & 1003.586 & 1001.814 & 999.858 \\
\hline 0.17089 & 1008.543 & 1007.407 & 1006.029 & 1004.436 & 1002.645 & 1000.673 \\
\hline 0.18048 & 1008.964 & 1007.815 & 1006.428 & 1004.825 & 1003.026 & 1001.042 \\
\hline 0.19721 & 1009.887 & 1008.720 & 1007.313 & 1005.695 & 1003.881 & 1001.884 \\
\hline
\end{tabular}




\begin{tabular}{|c|c|c|c|c|c|c|}
\hline \multicolumn{7}{|c|}{ Water + Glycine $(0.466 \mathrm{~m})+$ SDS } \\
\hline 0.00000 & 1014.209 & 1013.124 & 1011.805 & 1010.278 & 1008.557 & 1006.654 \\
\hline 0.00034 & 1014.250 & 1013.165 & 1011.846 & 1010.316 & 1008.596 & 1006.693 \\
\hline 0.00064 & 1014.257 & 1013.172 & 1011.851 & 1010.323 & 1008.601 & 1006.701 \\
\hline 0.01455 & 1014.949 & 1013.840 & 1012.501 & 1010.957 & 1009.220 & 1007.302 \\
\hline 0.02873 & 1015.490 & 1014.366 & 1013.014 & 1011.453 & 1009.700 & 1007.770 \\
\hline 0.04149 & 1015.973 & 1014.833 & 1013.469 & 1011.898 & 1010.136 & 1008.199 \\
\hline 0.05671 & 1016.607 & 1015.449 & 1014.066 & 1012.477 & 1010.704 & 1008.753 \\
\hline 0.07014 & 1017.317 & 1016.141 & 1014.742 & 1013.140 & 1011.330 & 1009.387 \\
\hline 0.08458 & 1017.871 & 1016.678 & 1015.263 & 1013.649 & 1011.848 & 1009.874 \\
\hline 0.09834 & 1018.414 & 1017.210 & 1015.776 & 1014.151 & 1012.337 & 1010.349 \\
\hline 0.11258 & 1019.000 & 1017.775 & 1016.332 & 1014.689 & 1012.863 & 1010.865 \\
\hline 0.12687 & 1019.540 & 1018.298 & 1016.837 & 1015.181 & 1013.344 & 1011.332 \\
\hline 0.14028 & 1020.096 & 1018.840 & 1017.367 & 1015.696 & 1013.847 & 1011.827 \\
\hline 0.15060 & 1020.458 & 1019.193 & 1017.708 & 1016.031 & 1014.171 & 1012.143 \\
\hline 0.16942 & 1021.156 & 1019.869 & 1018.366 & 1016.671 & 1014.797 & 1012.756 \\
\hline 0.18166 & 1021.545 & 1020.245 & 1018.732 & 1017.028 & 1015.143 & 1013.091 \\
\hline 0.19292 & 1021.958 & 1020.649 & 1019.122 & 1017.406 & 1015.551 & 1013.455 \\
\hline \multicolumn{7}{|c|}{ Water + DL-alanine $(0.049 \mathrm{~m})+\mathrm{SDS}$} \\
\hline 0.00000 & 1000.564 & 999.650 & 998.477 & 997.068 & 995.444 & 993.618 \\
\hline 0.00150 & 1000.723 & 999.807 & 998.627 & 997.212 & 995.583 & 993.757 \\
\hline 0.01442 & 1001.330 & 1000.393 & 999.196 & 997.766 & 996.124 & 994.283 \\
\hline 0.02820 & 1001.977 & 1001.025 & 999.794 & 998.366 & 996.709 & 994.860 \\
\hline 0.04313 & 1002.647 & 1001.674 & 1000.442 & 998.980 & 997.319 & 995.456 \\
\hline 0.05686 & 1003.314 & 1002.326 & 1001.079 & 999.607 & 997.925 & 996.051 \\
\hline 0.06819 & 1003.740 & 1002.735 & 1001.474 & 999.996 & 998.305 & 996.422 \\
\hline 0.08441 & 1004.468 & 1003.441 & 1002.161 & 1000.658 & 998.947 & 997.049 \\
\hline 0.09751 & 1005.058 & 1004.015 & 1002.720 & 1001.204 & 999.481 & 997.571 \\
\hline 0.11443 & 1005.717 & 1004.658 & 1003.348 & 1001.817 & 1000.082 & 998.158 \\
\hline 0.12739 & 1006.005 & 1004.909 & 1003.591 & 1002.048 & 1000.301 & 998.368 \\
\hline 0.13981 & 1006.551 & 1005.463 & 1004.127 & 1002.573 & 1000.814 & 998.870 \\
\hline 0.15325 & 1007.432 & 1006.323 & 1004.968 & 1003.399 & 1001.627 & 999.671 \\
\hline 0.16465 & 1007.977 & 1006.854 & 1005.485 & 1003.904 & 1002.121 & 1000.153 \\
\hline 0.17467 & 1008.264 & 1007.130 & 1005.756 & 1004.165 & 1002.376 & 1000.403 \\
\hline 0.19849 & 1009.288 & 1008.128 & 1006.731 & 1005.114 & 1003.302 & 1001.311 \\
\hline \multicolumn{7}{|c|}{ Water + DL-alanine $(0.438 \mathrm{~m})+\mathrm{SDS}$} \\
\hline 0.00000 & 1011.856 & 1010.828 & 1009.555 & 1008.064 & 1006.374 & 1004.498 \\
\hline 0.00082 & 1011.931 & 1010.902 & 1009.627 & 1008.135 & 1006.443 & 1004.566 \\
\hline 0.00156 & 1011.944 & 1010.914 & 1009.639 & 1008.146 & 1006.454 & 1004.576 \\
\hline 0.01554 & 1012.640 & 1011.589 & 1010.294 & 1008.789 & 1007.080 & 1005.185 \\
\hline 0.03025 & 1013.304 & 1012.229 & 1010.918 & 1009.379 & 1007.670 & 1005.770 \\
\hline 0.05630 & 1014.393 & 1013.290 & 1011.946 & 1010.399 & 1008.652 & 1006.726 \\
\hline 0.06954 & 1014.967 & 1013.844 & 1012.487 & 1010.923 & 1009.164 & 1007.226 \\
\hline 0.07950 & 1015.390 & 1014.253 & 1012.883 & 1011.302 & 1009.529 & 1007.593 \\
\hline 0.09943 & 1016.231 & 1015.060 & 1013.670 & 1012.050 & 1010.264 & 1008.318 \\
\hline 0.11285 & 1016.769 & 1015.580 & 1014.174 & 1012.541 & 1010.737 & 1008.780 \\
\hline 0.12791 & 1017.372 & 1016.161 & 1014.740 & 1013.080 & 1011.266 & 1009.297 \\
\hline 0.14159 & 1017.890 & 1016.659 & 1015.230 & 1013.550 & 1011.733 & 1009.754 \\
\hline 0.15603 & 1018.432 & 1017.208 & 1015.750 & 1014.080 & 1012.250 & 1010.243 \\
\hline 0.16924 & 1018.924 & 1017.683 & 1016.218 & 1014.560 & 1012.711 & 1010.693 \\
\hline 0.18305 & 1019.438 & 1018.179 & 1016.700 & 1015.020 & 1013.160 & 1011.140 \\
\hline 0.19867 & 1020.018 & 1018.745 & 1017.250 & 1015.558 & 1013.687 & 1011.645 \\
\hline
\end{tabular}

\section{TABLE-3}

ULTRASONIC VELOCITY $\left(\mathrm{u}, \mathrm{m} \mathrm{s}^{-1}\right.$ ) OF SDS IN AQUEOUS AND AQUEOUS AMINO ACID SOLUTIONS AT 288.15, 293.15, 298.15, 303.15, 308.15 AND 313.15 K, RESPECTIVELY

\begin{tabular}{|c|c|c|c|c|c|c|}
\hline $\mathrm{m}\left(\mathrm{mol} \mathrm{kg}{ }^{-1}\right)$ & $298.15 \mathrm{~K}$ & $303.15 \mathrm{~K}$ & $308.15 \mathrm{~K}$ & $313.15 \mathrm{~K}$ & $318.15 \mathrm{~K}$ & $323.15 \mathrm{~K}$ \\
\hline \multicolumn{7}{|c|}{ Water + SDS } \\
\hline 0.00000 & 1466.28 & 1482.58 & 1496.78 & 1509.18 & 1519.76 & 1528.78 \\
\hline 0.00034 & 1466.62 & 1482.86 & 1496.90 & 1509.40 & 1519.93 & 1528.99 \\
\hline 0.00089 & 1466.82 & 1482.86 & 1497.10 & 1509.49 & 1520.17 & 1529.22 \\
\hline 0.00136 & 1466.84 & 1482.93 & 1497.12 & 1509.52 & 1520.29 & 1529.28 \\
\hline 0.01499 & 1468.00 & 1483.60 & 1498.30 & 1510.70 & 1520.99 & 1529.90 \\
\hline 0.02999 & 1468.35 & 1484.70 & 1498.50 & 1510.52 & 1520.94 & 1529.66 \\
\hline 0.04398 & 1468.99 & 1484.82 & 1498.62 & 1510.49 & 1520.67 & 1529.29 \\
\hline 0.05807 & 1469.31 & 1484.97 & 1498.68 & 1510.34 & 1520.43 & 1528.92 \\
\hline
\end{tabular}




\begin{tabular}{|c|c|c|c|c|c|c|}
\hline 0.07199 & 1469.60 & 1485.08 & 1498.74 & 1510.23 & 1520.17 & 1528.53 \\
\hline 0.08594 & 1469.89 & 1485.24 & 1498.78 & 1510.14 & 1519.95 & 1528.19 \\
\hline 0.10085 & 1470.21 & 1485.43 & 1498.81 & 1510.04 & 1519.73 & 1527.83 \\
\hline 0.11498 & 1470.47 & 1485.58 & 1498.84 & 1509.97 & 1519.54 & 1527.52 \\
\hline 0.12860 & 1470.77 & 1485.73 & 1498.90 & 1509.89 & 1519.32 & 1527.19 \\
\hline 0.14257 & 1470.94 & 1485.89 & 1498.92 & 1509.83 & 1519.18 & 1526.96 \\
\hline 0.15700 & 1471.00 & 1486.03 & 1499.04 & 1509.78 & 1519.03 & 1526.72 \\
\hline 0.17194 & 1471.12 & 1486.27 & 1499.07 & 1509.62 & 1518.66 & 1526.14 \\
\hline 0.18610 & 1471.22 & 1486.34 & 1499.10 & 1509.44 & 1518.37 & 1525.73 \\
\hline 0.19845 & 1471.27 & 1486.53 & 1499.30 & 1509.37 & 1518.14 & 1525.39 \\
\hline \multicolumn{7}{|c|}{ Water + Glycine $(0.049 \mathrm{~m})+$ SDS } \\
\hline 0.00000 & 1469.23 & 1485.31 & 1499.43 & 1511.74 & 1522.32 & 1531.27 \\
\hline 0.00035 & 1469.40 & 1485.40 & 1499.49 & 1511.79 & 1522.44 & 1531.39 \\
\hline 0.00062 & 1469.43 & 1485.55 & 1499.54 & 1511.86 & 1522.49 & 1531.44 \\
\hline 0.01394 & 1471.59 & 1487.80 & 1501.10 & 1513.29 & 1523.70 & 1532.61 \\
\hline 0.02828 & 1471.80 & 1487.30 & 1501.14 & 1513.07 & 1523.36 & 1532.17 \\
\hline 0.04222 & 1471.86 & 1487.40 & 1501.11 & 1512.97 & 1523.18 & 1531.74 \\
\hline 0.05679 & 1472.10 & 1487.58 & 1501.13 & 1512.89 & 1522.96 & 1531.40 \\
\hline 0.07115 & 1472.40 & 1487.74 & 1501.15 & 1512.85 & 1522.73 & 1531.06 \\
\hline 0.08490 & 1472.70 & 1487.92 & 1501.21 & 1512.73 & 1522.55 & 1530.74 \\
\hline 0.09953 & 1473.00 & 1488.10 & 1501.26 & 1512.58 & 1522.31 & 1530.38 \\
\hline 0.11068 & 1473.16 & 1488.14 & 1501.19 & 1512.45 & 1522.06 & 1530.00 \\
\hline 0.12577 & 1473.47 & 1488.31 & 1501.22 & 1512.34 & 1521.77 & 1529.62 \\
\hline 0.14084 & 1473.82 & 1488.54 & 1501.30 & 1512.25 & 1521.57 & 1529.26 \\
\hline 0.14951 & 1474.03 & 1488.65 & 1501.35 & 1512.17 & 1521.47 & 1529.09 \\
\hline 0.17089 & 1474.50 & 1488.93 & 1501.41 & 1512.12 & 1521.15 & 1528.59 \\
\hline 0.18048 & 1474.71 & 1489.04 & 1501.45 & 1512.05 & 1520.98 & 1528.33 \\
\hline 0.19721 & 1475.00 & 1489.54 & 1501.79 & 1512.26 & 1521.06 & 1528.28 \\
\hline \multicolumn{7}{|c|}{ Water + Glycine $(0.466 \mathrm{~m})+\mathrm{SDS}$} \\
\hline 0.00000 & 1492.57 & 1507.85 & 1521.31 & 1532.99 & 1543.02 & 1551.50 \\
\hline 0.00034 & 1492.70 & 1508.00 & 1521.46 & 1533.14 & 1543.04 & 1551.53 \\
\hline 0.00064 & 1492.78 & 1508.05 & 1521.49 & 1533.16 & 1543.12 & 1551.62 \\
\hline 0.01455 & 1494.41 & 1509.46 & 1522.70 & 1534.20 & 1544.04 & 1552.87 \\
\hline 0.02873 & 1494.41 & 1509.36 & 1522.47 & 1533.86 & 1543.30 & 1552.10 \\
\hline 0.04149 & 1494.46 & 1509.30 & 1522.30 & 1533.56 & 1543.19 & 1551.87 \\
\hline 0.05671 & 1494.66 & 1509.35 & 1522.22 & 1533.35 & 1542.82 & 1551.29 \\
\hline 0.07014 & 1495.03 & 1509.59 & 1522.31 & 1533.28 & 1542.62 & 1550.72 \\
\hline 0.08458 & 1495.18 & 1509.62 & 1522.22 & 1533.07 & 1542.29 & 1550.50 \\
\hline 0.09834 & 1495.37 & 1509.67 & 1522.13 & 1532.84 & 1541.91 & 1549.90 \\
\hline 0.11258 & 1495.55 & 1509.72 & 1522.03 & 1532.61 & 1541.56 & 1549.71 \\
\hline 0.12687 & 1495.72 & 1509.74 & 1521.92 & 1532.35 & 1541.17 & 1548.88 \\
\hline 0.14028 & 1495.90 & 1509.81 & 1521.86 & 1532.18 & 1540.88 & 1548.37 \\
\hline 0.15060 & 1495.98 & 1509.80 & 1521.76 & 1531.98 & 1540.57 & 1547.70 \\
\hline 0.16942 & 1496.18 & 1509.81 & 1521.59 & 1531.64 & 1540.07 & 1547.00 \\
\hline 0.18166 & 1496.18 & 1509.71 & 1521.38 & 1531.33 & 1539.65 & 1546.49 \\
\hline 0.19292 & 1496.27 & 1509.67 & 1521.25 & 1531.10 & 1539.30 & 1546.02 \\
\hline \multicolumn{7}{|c|}{ Water + DL-alanine $(0.049 \mathrm{~m})+\mathrm{SDS}$} \\
\hline 0.00000 & 1469.87 & 1485.97 & 1499.99 & 1512.19 & 1522.74 & 1531.68 \\
\hline 0.00150 & 1470.50 & 1486.53 & 1500.58 & 1512.78 & 1523.37 & 1532.10 \\
\hline 0.01442 & 1472.73 & 1487.88 & 1501.76 & 1513.81 & 1524.77 & 1533.30 \\
\hline 0.02820 & 1472.93 & 1487.96 & 1501.74 & 1513.68 & 1524.10 & 1532.99 \\
\hline 0.04313 & 1472.51 & 1488.03 & 1501.64 & 1513.71 & 1523.74 & 1532.20 \\
\hline 0.05686 & 1473.02 & 1488.44 & 1501.98 & 1513.64 & 1523.66 & 1532.08 \\
\hline 0.06819 & 1472.70 & 1488.10 & 1502.03 & 1513.56 & 1523.48 & 1531.81 \\
\hline 0.08441 & 1472.98 & 1488.15 & 1501.39 & 1512.83 & 1522.57 & 1530.71 \\
\hline 0.09751 & 1473.26 & 1488.31 & 1501.43 & 1512.75 & 1522.35 & 1530.40 \\
\hline 0.11443 & 1473.82 & 1488.72 & 1501.74 & 1512.90 & 1522.40 & 1530.33 \\
\hline 0.12739 & 1473.40 & 1488.20 & 1501.05 & 1512.11 & 1521.52 & 1529.35 \\
\hline 0.13981 & 1473.55 & 1488.26 & 1501.07 & 1512.02 & 1521.32 & 1529.03 \\
\hline 0.15325 & 1474.60 & 1489.15 & 1501.78 & 1512.59 & 1521.74 & 1529.30 \\
\hline 0.16465 & 1474.82 & 1489.26 & 1501.80 & 1512.47 & 1521.50 & 1528.97 \\
\hline 0.17467 & 1474.95 & 1489.31 & 1501.74 & 1512.39 & 1521.35 & 1528.75 \\
\hline 0.19849 & 1474.99 & 1489.58 & 1501.76 & 1512.15 & 1520.91 & 1528.10 \\
\hline
\end{tabular}




\begin{tabular}{|c|c|c|c|c|c|c|}
\hline \multicolumn{7}{|c|}{ Water + DL-alanine $(0.438 \mathrm{~m})+\mathrm{SDS}$} \\
\hline 0.00000 & 1497.33 & 1512.32 & 1525.46 & 1536.85 & 1546.59 & 1554.80 \\
\hline 0.00082 & 1497.40 & 1512.38 & 1525.54 & 1536.94 & 1546.78 & 1554.88 \\
\hline 0.00156 & 1497.54 & 1512.50 & 1525.68 & 1537.17 & 1546.90 & 1554.94 \\
\hline 0.01554 & 1498.74 & 1513.42 & 1526.66 & 1537.97 & 1547.99 & 1555.14 \\
\hline 0.03025 & 1498.84 & 1513.38 & 1526.68 & 1537.69 & 1547.77 & 1554.80 \\
\hline 0.05630 & 1499.00 & 1513.36 & 1526.60 & 1537.27 & 1546.00 & 1554.20 \\
\hline 0.06954 & 1499.10 & 1513.48 & 1526.44 & 1536.99 & 1545.70 & 1553.75 \\
\hline 0.07950 & 1499.20 & 1513.53 & 1526.27 & 1536.80 & 1545.40 & 1553.35 \\
\hline 0.09943 & 1499.32 & 1513.38 & 1525.74 & 1536.27 & 1544.90 & 1552.14 \\
\hline 0.11285 & 1499.57 & 1513.48 & 1525.54 & 1535.87 & 1544.57 & 1551.77 \\
\hline 0.12791 & 1499.90 & 1513.66 & 1525.61 & 1535.75 & 1544.30 & 1551.37 \\
\hline 0.14159 & 1500.00 & 1513.64 & 1525.45 & 1535.47 & 1543.91 & 1550.84 \\
\hline 0.15603 & 1500.18 & 1513.68 & 1525.30 & 1535.21 & 1543.52 & 1550.31 \\
\hline 0.16924 & 1500.79 & 1514.15 & 1525.66 & 1535.40 & 1543.55 & 1550.22 \\
\hline 0.18305 & 1500.76 & 1513.99 & 1525.40 & 1534.95 & 1543.40 & 1549.45 \\
\hline 0.19867 & 1500.89 & 1513.96 & 1525.16 & 1534.70 & 1542.99 & 1548.95 \\
\hline
\end{tabular}

$$
\beta_{\mathrm{s}}=\frac{1}{\rho \mathrm{u}^{2}}
$$

where $\rho\left(\mathrm{kg} \mathrm{m}^{-3}\right)$ and $\beta_{\mathrm{s}}\left(\mathrm{Pa}^{-1}\right)$ are the density and adiabatic compressibility, respectively, of glucose and fructose in water. The calculated values of are presented in Table-4. And apparent molar adiabatic compressibility $\left(\phi_{\mathrm{K}}\right)$, was calculated from densities and data using eqn. 2 :

$$
\phi_{\mathrm{K}}=\frac{\beta_{\mathrm{S}} \rho_{\mathrm{o}}-\rho \beta_{\mathrm{S}}^{\mathrm{o}}}{\mathrm{m} \rho \rho_{\mathrm{o}}}+\frac{\beta_{\mathrm{S}} \mathrm{M}_{2}}{\rho}
$$

\begin{tabular}{|c|c|c|c|c|c|c|}
\hline \multicolumn{7}{|c|}{$\begin{array}{c}\text { TABLE-4 } \\
\text { ADIABATIC COMPRESSIBILITY }\left(\beta_{\mathrm{s}}, 10^{-10} \mathrm{~Pa}^{-1}\right) \text { OF SDS IN AQUEOUS AND AQUEOUS AMINO ACID } \\
\text { SOLUTIONS AT } 288.15,293.15,298.15,303.15,308.15 \text { AND } 313.15 \mathrm{~K} \text {, RESPECTIVELY }\end{array}$} \\
\hline $\mathrm{m}\left(\mathrm{mol} \mathrm{kg}^{-1}\right)$ & $298.15 \mathrm{~K}$ & $303.15 \mathrm{~K}$ & $308.15 \mathrm{~K}$ & $313.15 \mathrm{~K}$ & $318.15 \mathrm{~K}$ & $323.15 \mathrm{~K}$ \\
\hline \multicolumn{7}{|c|}{ Water + SDS } \\
\hline 0.00000 & 4.655 & 4.558 & 4.477 & 4.410 & 4.356 & 4.312 \\
\hline 0.00034 & 4.653 & 4.556 & 4.476 & 4.408 & 4.355 & 4.311 \\
\hline 0.00089 & 4.652 & 4.556 & 4.475 & 4.408 & 4.353 & 4.310 \\
\hline 0.00136 & 4.651 & 4.555 & 4.474 & 4.407 & 4.352 & 4.309 \\
\hline 0.01499 & 4.641 & 4.548 & 4.464 & 4.398 & 4.345 & 4.303 \\
\hline 0.02999 & 4.635 & 4.538 & 4.460 & 4.396 & 4.343 & 4.301 \\
\hline 0.04398 & 4.628 & 4.534 & 4.457 & 4.393 & 4.342 & 4.301 \\
\hline 0.05807 & 4.623 & 4.531 & 4.454 & 4.391 & 4.341 & 4.301 \\
\hline 0.07199 & 4.618 & 4.527 & 4.450 & 4.390 & 4.340 & 4.300 \\
\hline 0.08594 & 4.614 & 4.523 & 4.448 & 4.387 & 4.338 & 4.300 \\
\hline 0.10085 & 4.608 & 4.519 & 4.445 & 4.385 & 4.337 & 4.299 \\
\hline 0.11498 & 4.604 & 4.516 & 4.442 & 4.383 & 4.336 & 4.299 \\
\hline 0.12860 & 4.599 & 4.512 & 4.439 & 4.381 & 4.335 & 4.298 \\
\hline 0.14257 & 4.596 & 4.509 & 4.436 & 4.379 & 4.333 & 4.297 \\
\hline 0.15700 & 4.593 & 4.505 & 4.433 & 4.377 & 4.332 & 4.296 \\
\hline 0.17194 & 4.588 & 4.500 & 4.430 & 4.375 & 4.331 & 4.297 \\
\hline 0.18610 & 4.585 & 4.497 & 4.427 & 4.374 & 4.330 & 4.297 \\
\hline 0.19845 & 4.583 & 4.494 & 4.424 & 4.372 & 4.330 & 4.297 \\
\hline \multicolumn{7}{|c|}{ Water + Glycine $(0.049 \mathrm{~m})+$ SDS } \\
\hline 0.00000 & 4.629 & 4.534 & 4.454 & 4.388 & 4.334 & 4.292 \\
\hline 0.00035 & 4.628 & 4.533 & 4.453 & 4.387 & 4.333 & 4.291 \\
\hline 0.00062 & 4.628 & 4.532 & 4.453 & 4.387 & 4.333 & 4.290 \\
\hline 0.01394 & 4.611 & 4.515 & 4.441 & 4.376 & 4.323 & 4.281 \\
\hline 0.02828 & 4.606 & 4.515 & 4.438 & 4.374 & 4.323 & 4.281 \\
\hline 0.04222 & 4.603 & 4.512 & 4.435 & 4.372 & 4.321 & 4.281 \\
\hline 0.05679 & 4.599 & 4.508 & 4.432 & 4.370 & 4.320 & 4.280 \\
\hline 0.07115 & 4.593 & 4.504 & 4.429 & 4.368 & 4.318 & 4.280 \\
\hline 0.08490 & 4.589 & 4.500 & 4.426 & 4.366 & 4.317 & 4.279 \\
\hline 0.09953 & 4.584 & 4.496 & 4.423 & 4.364 & 4.316 & 4.279 \\
\hline 0.11068 & 4.581 & 4.494 & 4.422 & 4.363 & 4.315 & 4.279 \\
\hline 0.12577 & 4.576 & 4.490 & 4.419 & 4.361 & 4.315 & 4.279 \\
\hline 0.14084 & 4.571 & 4.486 & 4.416 & 4.359 & 4.313 & 4.278 \\
\hline 0.14951 & 4.568 & 4.483 & 4.414 & 4.358 & 4.312 & 4.278 \\
\hline 0.17089 & 4.561 & 4.478 & 4.410 & 4.354 & 4.310 & 4.277 \\
\hline 0.18048 & 4.557 & 4.475 & 4.408 & 4.353 & 4.310 & 4.277 \\
\hline 0.19721 & 4.551 & 4.468 & 4.402 & 4.348 & 4.306 & 4.273 \\
\hline
\end{tabular}




\begin{tabular}{|c|c|c|c|c|c|c|}
\hline \multicolumn{7}{|c|}{ Water + Glycine $(0.466 \mathrm{~m})+$ SDS } \\
\hline 0.00000 & 4.426 & 4.341 & 4.270 & 4.212 & 4.164 & 4.127 \\
\hline 0.00034 & 4.425 & 4.340 & 4.269 & 4.211 & 4.164 & 4.127 \\
\hline 0.00064 & 4.424 & 4.340 & 4.269 & 4.211 & 4.164 & 4.126 \\
\hline 0.01455 & 4.412 & 4.329 & 4.260 & 4.202 & 4.156 & 4.117 \\
\hline 0.02873 & 4.409 & 4.327 & 4.259 & 4.202 & 4.158 & 4.119 \\
\hline 0.04149 & 4.407 & 4.326 & 4.258 & 4.202 & 4.157 & 4.119 \\
\hline 0.05671 & 4.403 & 4.323 & 4.256 & 4.201 & 4.157 & 4.119 \\
\hline 0.07014 & 4.398 & 4.318 & 4.252 & 4.198 & 4.155 & 4.120 \\
\hline 0.08458 & 4.395 & 4.316 & 4.251 & 4.197 & 4.155 & 4.119 \\
\hline 0.09834 & 4.391 & 4.313 & 4.249 & 4.197 & 4.155 & 4.120 \\
\hline 0.11258 & 4.388 & 4.311 & 4.247 & 4.196 & 4.155 & 4.119 \\
\hline 0.12687 & 4.384 & 4.308 & 4.246 & 4.195 & 4.155 & 4.122 \\
\hline 0.14028 & 4.381 & 4.306 & 4.244 & 4.194 & 4.154 & 4.122 \\
\hline 0.15060 & 4.379 & 4.304 & 4.243 & 4.194 & 4.155 & 4.125 \\
\hline 0.16942 & 4.375 & 4.301 & 4.241 & 4.193 & 4.155 & 4.126 \\
\hline 0.18166 & 4.373 & 4.300 & 4.241 & 4.193 & 4.156 & 4.127 \\
\hline 0.19292 & 4.371 & 4.299 & 4.240 & 4.193 & 4.156 & 4.128 \\
\hline \multicolumn{7}{|c|}{ Water + DL-alanine $(0.049 \mathrm{~m})+\mathrm{SDS}$} \\
\hline 0.00000 & 4.626 & 4.530 & 4.451 & 4.386 & 4.332 & 4.290 \\
\hline 0.00150 & 4.621 & 4.526 & 4.447 & 4.382 & 4.328 & 4.287 \\
\hline 0.01442 & 4.604 & 4.515 & 4.438 & 4.373 & 4.318 & 4.278 \\
\hline 0.02820 & 4.600 & 4.512 & 4.435 & 4.372 & 4.319 & 4.277 \\
\hline 0.04313 & 4.600 & 4.509 & 4.433 & 4.369 & 4.319 & 4.279 \\
\hline 0.05686 & 4.594 & 4.503 & 4.428 & 4.366 & 4.316 & 4.277 \\
\hline 0.06819 & 4.594 & 4.503 & 4.426 & 4.365 & 4.316 & 4.277 \\
\hline 0.08441 & 4.588 & 4.500 & 4.427 & 4.367 & 4.318 & 4.281 \\
\hline 0.09751 & 4.584 & 4.496 & 4.424 & 4.365 & 4.317 & 4.280 \\
\hline 0.11443 & 4.578 & 4.491 & 4.419 & 4.361 & 4.314 & 4.278 \\
\hline 0.12739 & 4.579 & 4.493 & 4.422 & 4.365 & 4.318 & 4.282 \\
\hline 0.13981 & 4.575 & 4.490 & 4.420 & 4.363 & 4.317 & 4.282 \\
\hline 0.15325 & 4.565 & 4.481 & 4.412 & 4.356 & 4.311 & 4.277 \\
\hline 0.16465 & 4.561 & 4.478 & 4.410 & 4.354 & 4.311 & 4.277 \\
\hline 0.17467 & 4.559 & 4.477 & 4.409 & 4.354 & 4.310 & 4.277 \\
\hline 0.19849 & 4.554 & 4.471 & 4.404 & 4.351 & 4.309 & 4.277 \\
\hline \multicolumn{7}{|c|}{ Water + DL-alanine $(0.438 \mathrm{~m})+\mathrm{SDS}$} \\
\hline 0.00000 & 4.408 & 4.325 & 4.257 & 4.200 & 4.154 & 4.118 \\
\hline 0.00082 & 4.407 & 4.325 & 4.256 & 4.199 & 4.153 & 4.117 \\
\hline 0.00156 & 4.406 & 4.324 & 4.255 & 4.198 & 4.152 & 4.117 \\
\hline 0.01554 & 4.396 & 4.316 & 4.247 & 4.191 & 4.144 & 4.114 \\
\hline 0.03025 & 4.393 & 4.313 & 4.244 & 4.190 & 4.143 & 4.113 \\
\hline 0.05630 & 4.387 & 4.309 & 4.240 & 4.188 & 4.148 & 4.112 \\
\hline 0.06954 & 4.384 & 4.306 & 4.239 & 4.187 & 4.148 & 4.113 \\
\hline 0.07950 & 4.382 & 4.304 & 4.238 & 4.187 & 4.148 & 4.113 \\
\hline 0.09943 & 4.377 & 4.301 & 4.238 & 4.187 & 4.147 & 4.117 \\
\hline 0.11285 & 4.374 & 4.299 & 4.237 & 4.187 & 4.147 & 4.117 \\
\hline 0.12791 & 4.369 & 4.295 & 4.234 & 4.185 & 4.146 & 4.117 \\
\hline 0.14159 & 4.366 & 4.293 & 4.233 & 4.185 & 4.147 & 4.118 \\
\hline 0.15603 & 4.363 & 4.291 & 4.232 & 4.184 & 4.147 & 4.118 \\
\hline 0.16924 & 4.357 & 4.286 & 4.228 & 4.181 & 4.145 & 4.117 \\
\hline 0.18305 & 4.355 & 4.285 & 4.227 & 4.182 & 4.143 & 4.119 \\
\hline 0.19867 & 4.352 & 4.283 & 4.226 & 4.181 & 4.144 & 4.120 \\
\hline
\end{tabular}

where, $\mathrm{m}\left(\mathrm{mol} \mathrm{kg}^{-1}\right)=$ molality of SDS in binary or ternary system, $\rho\left(\mathrm{kg} \mathrm{m}^{-3}\right)=$ density of SDS in binary or ternary system, $\beta_{\mathrm{S}}\left(\mathrm{Pa}^{-1}\right)=$ adiabatic compressibility of SDS in binary or ternary system, $\rho_{0}\left(\mathrm{~kg} \mathrm{~m}^{-3}\right)=$ density of SDS in water, $\beta_{\mathrm{S}}^{\circ}\left(\mathrm{Pa}^{-1}\right)=$ adiabatic compressibility of SDS in water and $\mathrm{M}_{2}\left(\mathrm{~kg} \mathrm{~mol}^{-1}\right)$ $=$ molar mass of SDS.

The $\phi_{\mathrm{K}}$ values was listed in Table-5 and graphically represented in Fig. 1(a) for water and in Fig. 1(b) for each system (at temperature). For each system, the magnitude of $\phi_{\mathrm{K}}$ increases with concentration of SDS. Due to the relaxation of water molecules (as monomeric form) from the hydration sphere of co-sphere of SDS and amino acids to bulk caused by the interactions between them, $\phi_{\mathrm{K}}$ increases. The water molecules in the monomeric form are more compressible [27,28]. From figure, it is observed that at lower concentration zone $\phi_{\mathrm{K}}$ increases rapidly after that $\phi_{\mathrm{K}}$ shows very slow increasing trend with the increase in concentration of SDS. So, there appeared a definite break point called CMC. After CMC, slow increasing trend reveals that $\mathrm{CMC}$ formation render the relaxation of water molecules to bulk.

Critical micelle concentration (CMC): The CMC's of SDS in aqueous and aqueous amino acid solutions was deter- 


\begin{tabular}{|c|c|c|c|c|c|c|}
\hline \multicolumn{7}{|c|}{$\begin{array}{c}\text { TABLE-5 } \\
\text { APPARENT MOLAR ADIABATIC COMPRESSIBILITY }\left(\phi_{\mathrm{K}}, 10^{-14} \mathrm{~m}^{3} \mathrm{~mol}^{-1} \mathrm{~Pa}^{-1}\right) \text { OF SDS IN AQUEOUS AND AQUEOUS }\end{array}$} \\
\hline $\mathrm{m}\left(\mathrm{mol} \mathrm{kg}^{-1}\right)$ & $298.15 \mathrm{~K}$ & $303.15 \mathrm{~K}$ & $308.15 \mathrm{~K}$ & $313.15 \mathrm{~K}$ & $318.15 \mathrm{~K}$ & $323.15 \mathrm{~K}$ \\
\hline \multicolumn{7}{|c|}{ Water + SDS } \\
\hline 0.00034 & -60.2 & -46.4 & -17.5 & -33.8 & -24.2 & -30.5 \\
\hline 0.00089 & -32.4 & -13.0 & -15.1 & -13.8 & -20.0 & -21.3 \\
\hline 0.00136 & -18.7 & -8.2 & -7.3 & -7.0 & -14.7 & -13.0 \\
\hline 0.01499 & 1.0 & 4.1 & 2.2 & 2.3 & 3.6 & 4.1 \\
\hline 0.02999 & 4.2 & 4.3 & 5.1 & 6.0 & 6.3 & 6.9 \\
\hline 0.04398 & 4.8 & 5.6 & 6.2 & 6.9 & 7.5 & 8.0 \\
\hline 0.05807 & 5.5 & 6.2 & 6.8 & 7.6 & 8.1 & 8.6 \\
\hline 0.07199 & 5.9 & 6.7 & 7.1 & 7.9 & 8.4 & 9.0 \\
\hline 0.08594 & 6.2 & 6.9 & 7.4 & 8.1 & 8.7 & 9.2 \\
\hline 0.10085 & 6.4 & 7.1 & 7.6 & 8.3 & 8.8 & 9.4 \\
\hline 0.11498 & 6.6 & 7.3 & 7.8 & 8.4 & 9.0 & 9.5 \\
\hline 0.12860 & 6.7 & 7.4 & 7.9 & 8.5 & 9.1 & 9.6 \\
\hline 0.14257 & 6.9 & 7.5 & 8.0 & 8.6 & 9.1 & 9.6 \\
\hline 0.15700 & 7.1 & 7.6 & 8.0 & 8.7 & 9.2 & 9.7 \\
\hline 0.17194 & 7.1 & 7.6 & 8.0 & 8.7 & 9.2 & 9.8 \\
\hline 0.18610 & 7.3 & 7.7 & 8.1 & 8.8 & 9.3 & 9.9 \\
\hline 0.19845 & 7.4 & 7.7 & 8.2 & 8.9 & 9.4 & 9.9 \\
\hline \multicolumn{7}{|c|}{ Water + Glycine $(0.049 \mathrm{~m})+$ SDS } \\
\hline 0.00035 & -28.6 & -13.6 & -7.6 & $\begin{array}{c}-5.4 \\
\end{array}$ & -16.7 & -17.0 \\
\hline 0.00062 & -16.0 & -19.3 & -5.7 & -6.6 & -10.6 & -10.3 \\
\hline 0.01394 & -2.7 & -2.9 & 0.9 & 1.5 & 2.4 & 2.6 \\
\hline 0.02828 & 2.9 & 4.3 & 5.0 & 5.8 & 6.4 & 6.8 \\
\hline 0.04222 & 4.7 & 5.6 & 6.2 & 6.9 & 7.4 & 7.9 \\
\hline 0.05679 & 5.6 & 6.3 & 6.9 & 7.5 & 8.1 & 8.6 \\
\hline 0.07115 & 6.0 & 6.7 & 7.3 & 7.8 & 8.4 & 8.9 \\
\hline 0.08490 & 6.2 & 6.9 & 7.5 & 8.0 & 8.6 & 9.1 \\
\hline 0.09953 & 6.5 & 7.1 & 7.7 & 8.3 & 8.8 & 9.3 \\
\hline 0.11068 & 6.7 & 7.3 & 7.9 & 8.4 & 8.9 & 9.5 \\
\hline 0.12577 & 6.8 & 7.4 & 8.0 & 8.6 & 9.1 & 9.6 \\
\hline 0.14084 & 6.9 & 7.5 & 8.1 & 8.6 & 9.1 & 9.7 \\
\hline 0.14951 & 6.9 & 7.5 & 8.1 & 8.6 & 9.1 & 9.7 \\
\hline 0.17089 & 7.0 & 7.6 & 8.2 & 8.7 & 9.2 & 9.7 \\
\hline 0.18048 & 7.0 & 7.6 & 8.2 & 8.7 & 9.3 & 9.8 \\
\hline 0.19721 & 7.0 & 7.5 & 8.0 & 8.6 & 9.1 & 9.6 \\
\hline \multicolumn{7}{|c|}{ Water + Glycine $(0.466 \mathrm{~m})+$ SDS } \\
\hline 0.00034 & -20.4 & -23.2 & -22.6 & -21.4 & -0.7 & -2.3 \\
\hline 0.00064 & -12.9 & -11.6 & -9.3 & -8.1 & -2.0 & -4.0 \\
\hline 0.01455 & 0.8 & 1.9 & 2.9 & 3.6 & 4.4 & 3.2 \\
\hline 0.02873 & 5.0 & 5.7 & 6.4 & 7.0 & 8.1 & 7.5 \\
\hline 0.04149 & 6.2 & 6.9 & 7.5 & 8.0 & 8.6 & 8.3 \\
\hline 0.05671 & 6.7 & 7.3 & 7.9 & 8.5 & 9.0 & 9.0 \\
\hline 0.07014 & 6.7 & 7.3 & 7.8 & 8.4 & 8.9 & 9.2 \\
\hline 0.08458 & 7.0 & 7.5 & 8.1 & 8.6 & 9.2 & 9.3 \\
\hline 0.09834 & 7.2 & 7.7 & 8.3 & 8.8 & 9.3 & 9.6 \\
\hline 0.11258 & 7.3 & 7.8 & 8.4 & 8.9 & 9.4 & 9.6 \\
\hline 0.12687 & 7.4 & 8.0 & 8.5 & 9.0 & 9.6 & 9.9 \\
\hline 0.14028 & 7.5 & 8.0 & 8.6 & 9.1 & 9.6 & 10.0 \\
\hline 0.15060 & 7.6 & 8.1 & 8.6 & 9.2 & 9.7 & 10.2 \\
\hline 0.16942 & 7.7 & 8.2 & 8.7 & 9.3 & 9.8 & 10.3 \\
\hline 0.18166 & 7.8 & 8.3 & 8.9 & 9.4 & 9.9 & 10.4 \\
\hline 0.19292 & 7.8 & 8.4 & 8.9 & 9.4 & 9.9 & 10.4 \\
\hline \multicolumn{7}{|c|}{ Water + DL-alanine $(0.049 \mathrm{~m})+\mathrm{SDS}$} \\
\hline 0.00150 & -22.9 & -19.2 & -19.5 & -18.7 & -19.6 & -11.4 \\
\hline 0.01442 & -4.0 & 0.3 & 1.1 & 1.9 & 0.4 & 2.1 \\
\hline 0.02820 & 1.9 & 4.3 & 5.0 & 5.5 & 5.9 & 6.0 \\
\hline 0.04313 & 5.0 & 5.9 & 6.5 & 6.7 & 7.4 & 8.0 \\
\hline 0.05686 & 5.3 & 6.1 & 6.6 & 7.2 & 7.8 & 8.3 \\
\hline 0.06819 & 6.3 & 7.0 & 7.1 & 7.7 & 8.2 & 8.7 \\
\hline 0.08441 & 6.7 & 7.3 & 7.9 & 8.4 & 9.0 & 9.5 \\
\hline 0.09751 & 6.8 & 7.5 & 8.0 & 8.5 & 9.1 & 9.6 \\
\hline 0.11443 & 6.9 & 7.5 & 8.1 & 8.6 & 9.1 & 9.6 \\
\hline
\end{tabular}




\begin{tabular}{|c|c|c|c|c|c|c|}
\hline 0.12739 & 7.5 & 8.1 & 8.7 & 9.2 & 9.7 & 10.2 \\
\hline 0.13981 & 7.6 & 8.2 & 8.7 & 9.2 & 9.7 & 10.2 \\
\hline 0.15325 & 7.1 & 7.7 & 8.3 & 8.8 & 9.3 & 9.8 \\
\hline 0.16465 & 7.1 & 7.7 & 8.3 & 8.8 & 9.3 & 9.9 \\
\hline 0.17467 & 7.2 & 7.9 & 8.4 & 8.9 & 9.4 & 9.9 \\
\hline 0.19849 & 7.4 & 7.9 & 8.5 & 9.0 & 9.5 & 10.0 \\
\hline \multicolumn{7}{|c|}{ Water + DL-alanine $(0.438 \mathrm{~m})+\mathrm{SDS}$} \\
\hline 0.00082 & -0.2 & 0.6 & -0.5 & -1.0 & -7.3 & 0.0 \\
\hline 0.00156 & -0.1 & 1.1 & -0.1 & -3.5 & -2.9 & 3.0 \\
\hline 0.01554 & 2.9 & 4.2 & 3.9 & 4.3 & 3.4 & 7.1 \\
\hline 0.03025 & 5.5 & 6.4 & 6.2 & 6.9 & 6.3 & 8.4 \\
\hline 0.05630 & 6.9 & 7.6 & 7.5 & 8.2 & 9.1 & 9.1 \\
\hline 0.06954 & 7.2 & 7.7 & 7.8 & 8.5 & 9.3 & 9.4 \\
\hline 0.07950 & 7.3 & 7.8 & 8.0 & 8.6 & 9.4 & 9.6 \\
\hline 0.09943 & 7.5 & 8.1 & 8.5 & 9.0 & 9.6 & 10.1 \\
\hline 0.11285 & 7.6 & 8.1 & 8.6 & 9.2 & 9.7 & 10.1 \\
\hline 0.12791 & 7.6 & 8.1 & 8.6 & 9.2 & 9.7 & 10.1 \\
\hline 0.14159 & 7.7 & 8.2 & 8.7 & 9.3 & 9.8 & 10.2 \\
\hline 0.15603 & 7.7 & 8.3 & 8.8 & 9.3 & 9.8 & 10.3 \\
\hline 0.16924 & 7.6 & 8.2 & 8.7 & 9.2 & 9.7 & 10.2 \\
\hline 0.18305 & 7.7 & 8.3 & 8.8 & 9.3 & 9.7 & 10.4 \\
\hline 0.19867 & 7.8 & 8.4 & 8.9 & 9.4 & 9.8 & 10.4 \\
\hline
\end{tabular}
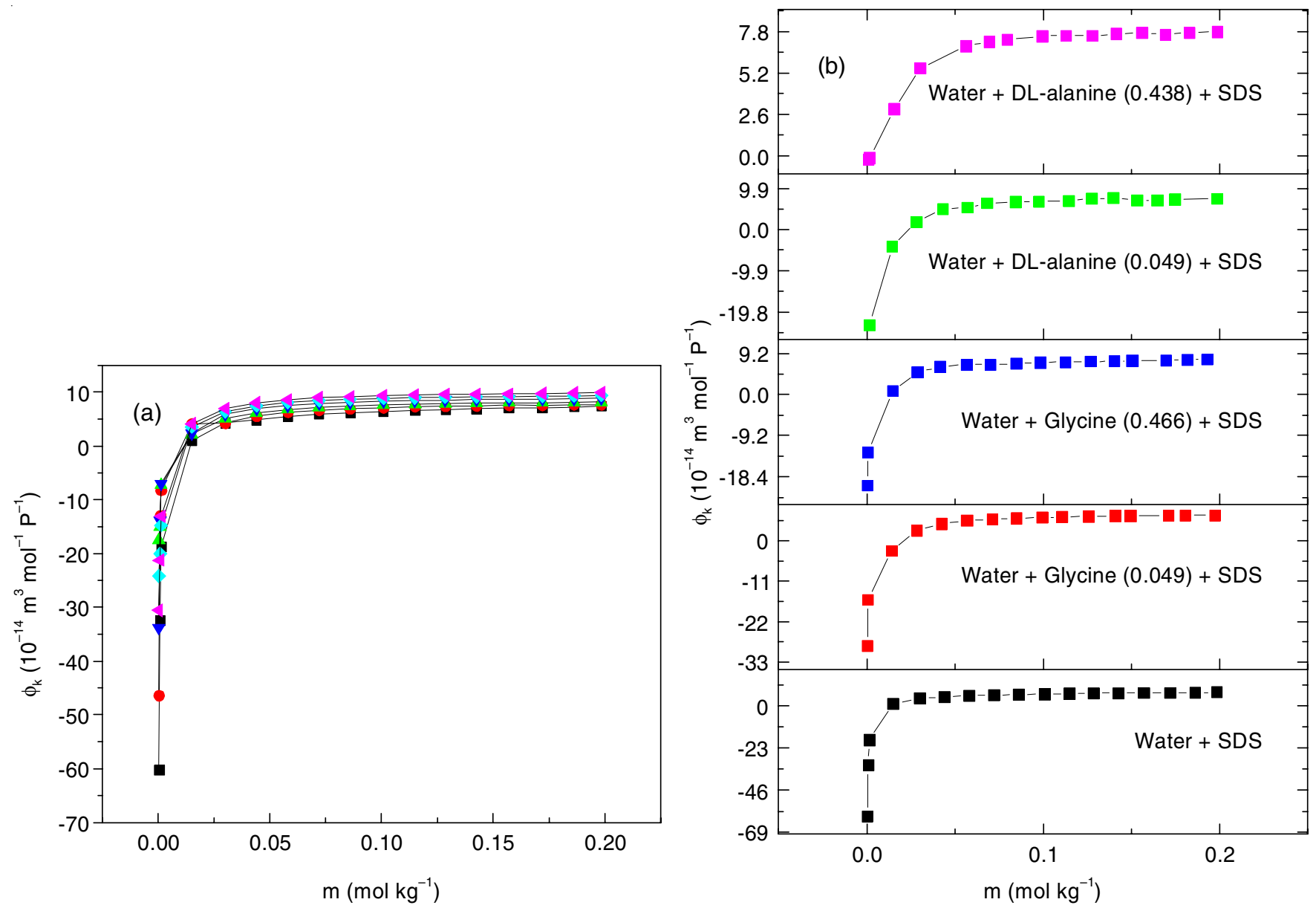

Fig. 1. Apparent molar adiabatic compressibility ( $\left.\phi_{\mathrm{K}}\right)$ of SDS in (a) in water as a function of molality at different temperatures; $288.15 \mathrm{~K}$, $293.15 \mathrm{~K}$, $\triangle 298.15 \mathrm{~K}, \nabla 303.15 \mathrm{~K}, \diamond 308.15 \mathrm{~K}, \triangleleft 313.15 \mathrm{~K}$. (b) in aqueous and aqueous amino acid solutions at $288.15 \mathrm{~K}$

mined using $\phi_{\mathrm{K}}$ data by extrapolation method (Table-6) shown in Fig. 2. It is observed that CMC values of SDS in aqueous solution are larger than the previous literature values $(\sim 0.009)$ [29]. And these values with the concentration of aqueous amino acid solution also larger than the aqueous solution indicate that glycine and alanine are water structure breakers. The addition of structure breakers causes the CMC increasing [30]. Critical micelle concentration values are not regular with temperature may be due to uncertainties association to low region concentration of SDS. SDS contains $\mathrm{CH}_{3}-\left(\mathrm{CH}_{2}\right)_{11^{-}}, \mathrm{SO}_{4}{ }^{2-}$ and amino acids have $\mathrm{NH}_{3}{ }^{+}, \mathrm{COO}^{-}$and hydrophobic group. Micelle formation occurs due to the closeness of $\mathrm{CH}_{3}-\left(\mathrm{CH}_{2}\right)$ - group of SDS. 


\begin{tabular}{|c|c|c|c|c|c|c|}
\hline \multicolumn{7}{|c|}{$\begin{array}{c}\text { TABLE-6 } \\
\text { CMC }\left(\mathrm{mol} \mathrm{kg}^{-1}\right) \text { OF SDS IN AQUEOUS AND AQUEOUS AMINO ACID SOLUTIONS } \\
\text { AT } 288.15,293.15,298.15,303.15,308.15 \text { AND } 313.15 \text { K RESPECTIVELY }\end{array}$} \\
\hline System & $298.15 \mathrm{~K}$ & $303.15 \mathrm{~K}$ & $308.15 \mathrm{~K}$ & $313.15 \mathrm{~K}$ & $318.15 \mathrm{~K}$ & $323.15 \mathrm{~K}$ \\
\hline Water + SDS & 0.01477 & 0.01457 & 0.01357 & 0.01357 & 0.01357 & 0.01407 \\
\hline Water + Glycine $(0.049)+$ SDS & 0.02449 & 0.02449 & 0.02203 & 0.02203 & 0.02278 & 0.02278 \\
\hline Water + Glycine $(0.466)+$ SDS & 0.02626 & 0.02626 & 0.02727 & 0.02727 & 0.02558 & 0.02821 \\
\hline Water + DL-alanine $(0.049)+$ SDS & 0.02449 & 0.02449 & 0.02203 & 0.02203 & 0.02278 & 0.02278 \\
\hline Water + DL-alanine $(0.438)+$ SDS & 0.03795 & 0.03626 & 0.03874 & 0.03425 & 0.03535 & 0.03767 \\
\hline
\end{tabular}

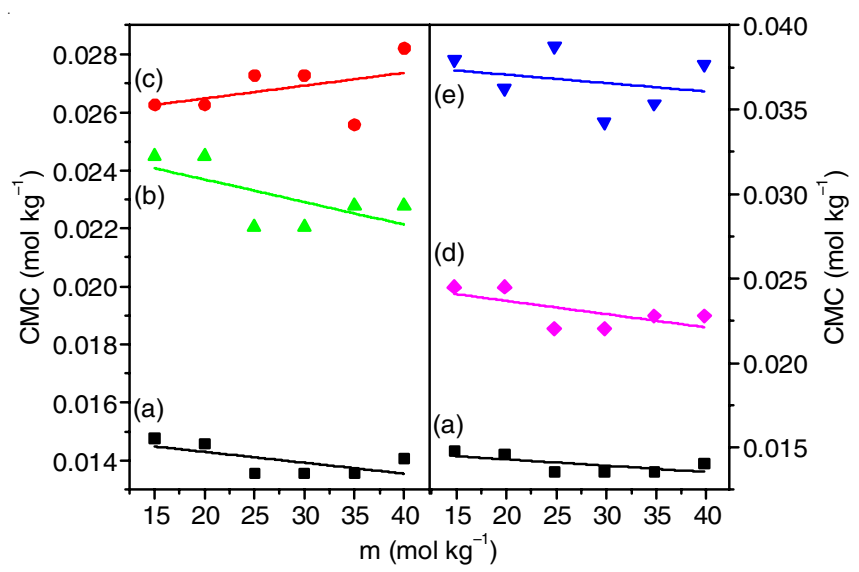

Fig. 2. Critical micelle concentration (CMC) of SDS in (a) water (b) $\Delta$ $0.049 \mathrm{~m}$ glycine (c) $\bullet 0.466 \mathrm{~m}$ glycine $(\mathrm{d}) \bullet 0.049 \mathrm{~m}$ DL-alanine (e) $\boldsymbol{\nabla} 0.438 \mathrm{~m}$ DL-alanine as a function of molality at different temperatures

With the increasing concentration of amino acids in SDS solution, repulsion between $\mathrm{COO}^{-}$(amino acid) and $\mathrm{SO}_{4}{ }^{2-}$ (SDS) groups causes the increasing distance between SDS molecules. So, for this reason CMC also increases.

Acoustic impedance (Z): The acoustic impedance (Z) was calculated from the experimental density and ultrasonic velocity using eqn. 3 :

$$
\mathrm{Z}=\mathrm{u} \rho
$$

Acoustic impedance of SDS in water and in aqueous amino acid solution is presented in Table-7 and shown in Figs.
3 and 4. Fig. 3 demonstrated that acoustic impedance increases with the increase molality of SDS. Acoustic impedance measure resistance to propagation of sound wave through any medium. The increase in $\mathrm{Z}$ with molality of SDS indicates that as concentration increases the sound wave as to face more resistance to flow. As the concentration increases solute-solute interaction increases. From Fig. 4, it is observed that acoustic impedances of SDS in DL-alanine are slightly greater from in glycine due to having an extra $\mathrm{CH}_{3}$ - group of DL-alanine. And

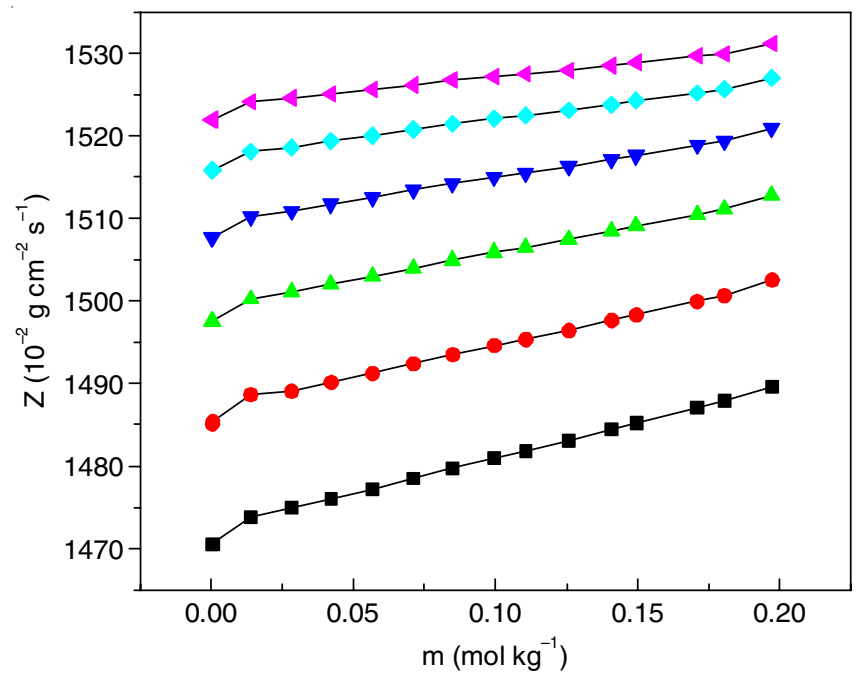

Fig. 3. Acoustic impedance (Z) of SDS in $0.049 \mathrm{~m}$ glycine as a function of molality at different temperatures; $288.15 \mathrm{~K}$, $293.15 \mathrm{~K}$, A $298.15 \mathrm{~K}, \boldsymbol{\nabla} 303.15 \mathrm{~K}, \diamond 308.15 \mathrm{~K}, \triangleleft 313.15 \mathrm{~K}$

TABLE-7

ACOUSTIC IMPEDANCE $\left(\mathrm{Z}, 10^{-2} \mathrm{~g} \mathrm{~cm}^{-2} \mathrm{~s}^{-1}\right)$ OF SDS IN AQUEOUS AND AQUEOUS AMINO ACID SOLUTIONS AT 288.15, 293.15, 298.15, 303.15, 308.15 AND 313.15 K, RESPECTIVELY

\begin{tabular}{|c|c|c|c|c|c|c|}
\hline $\mathrm{m}\left(\mathrm{mol} \mathrm{kg}{ }^{-1}\right)$ & $298.15 \mathrm{~K}$ & $303.15 \mathrm{~K}$ & $308.15 \mathrm{~K}$ & $313.15 \mathrm{~K}$ & $318.15 \mathrm{~K}$ & $323.15 \mathrm{~K}$ \\
\hline \multicolumn{7}{|c|}{ Water + SDS } \\
\hline 0.00034 & 1465.36 & 1480.25 & 1492.53 & 1502.88 & 1510.91 & 1517.13 \\
\hline 0.00089 & 1465.60 & 1480.30 & 1492.77 & 1503.01 & 1511.19 & 1517.40 \\
\hline 0.00136 & 1465.65 & 1480.39 & 1492.81 & 1503.07 & 1511.33 & 1517.49 \\
\hline 0.01499 & 1467.90 & 1482.13 & 1495.04 & 1505.26 & 1513.03 & 1519.09 \\
\hline 0.02999 & 1469.29 & 1484.25 & 1496.24 & 1506.07 & 1513.95 & 1519.81 \\
\hline 0.04398 & 1470.89 & 1485.31 & 1497.29 & 1506.96 & 1514.59 & 1520.33 \\
\hline 0.05807 & 1472.16 & 1486.39 & 1498.27 & 1507.71 & 1515.23 & 1520.83 \\
\hline 0.07199 & 1473.39 & 1487.43 & 1499.23 & 1508.49 & 1515.85 & 1521.30 \\
\hline 0.08594 & 1474.62 & 1488.51 & 1500.18 & 1509.29 & 1516.50 & 1521.83 \\
\hline 0.10085 & 1475.94 & 1489.68 & 1501.16 & 1510.13 & 1517.21 & 1522.38 \\
\hline 0.11498 & 1477.08 & 1490.69 & 1502.05 & 1510.90 & 1517.85 & 1522.88 \\
\hline 0.12860 & 1478.26 & 1491.71 & 1502.96 & 1511.65 & 1518.44 & 1523.36 \\
\hline 0.14257 & 1479.28 & 1492.70 & 1503.79 & 1512.40 & 1519.10 & 1523.91 \\
\hline 0.15700 & 1480.26 & 1493.74 & 1504.80 & 1513.22 & 1519.80 & 1524.50 \\
\hline 0.17194 & 1481.69 & 1495.12 & 1505.94 & 1514.13 & 1520.48 & 1524.95 \\
\hline 0.18610 & 1482.48 & 1495.95 & 1506.72 & 1514.70 & 1520.93 & 1525.28 \\
\hline 0.19845 & 1483.15 & 1496.81 & 1507.58 & 1515.28 & 1521.34 & 1525.57 \\
\hline
\end{tabular}




\begin{tabular}{|c|c|c|c|c|c|c|}
\hline \multicolumn{7}{|c|}{ Water + Glycine $(0.049 \mathrm{~m})+$ SDS } \\
\hline 0.00035 & 1470.52 & 1485.17 & 1497.48 & 1507.63 & 1515.77 & 1521.88 \\
\hline 0.00062 & 1470.58 & 1485.34 & 1497.56 & 1507.73 & 1515.84 & 1521.95 \\
\hline 0.01394 & 1473.84 & 1488.67 & 1500.17 & 1510.19 & 1518.07 & 1524.13 \\
\hline 0.02828 & 1474.97 & 1489.08 & 1501.11 & 1510.85 & 1518.60 & 1524.54 \\
\hline 0.04222 & 1476.04 & 1490.17 & 1502.06 & 1511.71 & 1519.37 & 1525.05 \\
\hline 0.05679 & 1477.20 & 1491.25 & 1502.96 & 1512.51 & 1520.01 & 1525.56 \\
\hline 0.07115 & 1478.53 & 1492.43 & 1503.98 & 1513.45 & 1520.75 & 1526.17 \\
\hline 0.08490 & 1479.76 & 1493.52 & 1504.94 & 1514.21 & 1521.43 & 1526.71 \\
\hline 0.09953 & 1480.99 & 1494.61 & 1505.88 & 1514.94 & 1522.06 & 1527.20 \\
\hline 0.11068 & 1481.84 & 1495.32 & 1506.47 & 1515.45 & 1522.44 & 1527.43 \\
\hline 0.12577 & 1483.10 & 1496.43 & 1507.45 & 1516.24 & 1523.03 & 1527.92 \\
\hline 0.14084 & 1484.46 & 1497.65 & 1508.46 & 1517.10 & 1523.77 & 1528.48 \\
\hline 0.14951 & 1485.26 & 1498.34 & 1509.09 & 1517.59 & 1524.23 & 1528.87 \\
\hline 0.17089 & 1487.10 & 1499.96 & 1510.46 & 1518.83 & 1525.17 & 1529.62 \\
\hline 0.18048 & 1487.93 & 1500.68 & 1511.10 & 1519.35 & 1525.58 & 1529.92 \\
\hline 0.19721 & 1489.58 & 1502.53 & 1512.77 & 1520.87 & 1526.96 & 1531.16 \\
\hline \multicolumn{7}{|c|}{ Water + Glycine $(0.466 \mathrm{~m})+$ SDS } \\
\hline 0.00034 & 1513.97 & 1527.85 & 1539.48 & 1548.96 & 1556.30 & 1561.91 \\
\hline 0.00064 & 1514.06 & 1527.91 & 1539.52 & 1548.99 & 1556.39 & 1562.02 \\
\hline 0.01455 & 1516.75 & 1530.35 & 1541.74 & 1551.01 & 1558.28 & 1564.21 \\
\hline 0.02873 & 1517.56 & 1531.04 & 1542.28 & 1551.43 & 1558.27 & 1564.16 \\
\hline 0.04149 & 1518.33 & 1531.69 & 1542.80 & 1551.81 & 1558.83 & 1564.59 \\
\hline 0.05671 & 1519.48 & 1532.67 & 1543.63 & 1552.48 & 1559.33 & 1564.87 \\
\hline 0.07014 & 1520.92 & 1533.96 & 1544.75 & 1553.43 & 1560.10 & 1565.28 \\
\hline 0.08458 & 1521.90 & 1534.80 & 1545.45 & 1553.99 & 1560.56 & 1565.81 \\
\hline 0.09834 & 1522.91 & 1535.65 & 1546.14 & 1554.53 & 1560.93 & 1565.94 \\
\hline 0.11258 & 1523.97 & 1536.56 & 1546.89 & 1555.12 & 1561.39 & 1566.55 \\
\hline 0.12687 & 1524.95 & 1537.37 & 1547.54 & 1555.61 & 1561.74 & 1566.43 \\
\hline 0.14028 & 1525.96 & 1538.25 & 1548.29 & 1556.23 & 1562.22 & 1566.68 \\
\hline 0.15060 & 1526.58 & 1538.78 & 1548.71 & 1556.54 & 1562.40 & 1566.49 \\
\hline 0.16942 & 1527.83 & 1539.81 & 1549.54 & 1557.17 & 1562.86 & 1566.73 \\
\hline 0.18166 & 1528.42 & 1540.27 & 1549.88 & 1557.41 & 1562.96 & 1566.74 \\
\hline 0.19292 & 1529.13 & 1540.84 & 1550.34 & 1557.75 & 1563.24 & 1566.82 \\
\hline \multicolumn{7}{|c|}{ Water + DL-alanine $(0.049 \mathrm{~m})+\mathrm{SDS}$} \\
\hline 0.00150 & 1471.56 & 1486.24 & 1498.52 & 1508.56 & 1516.64 & 1522.54 \\
\hline 0.01442 & 1474.69 & 1488.46 & 1500.55 & 1510.43 & 1518.86 & 1524.53 \\
\hline 0.02820 & 1475.84 & 1489.49 & 1501.43 & 1511.21 & 1519.08 & 1525.11 \\
\hline 0.04313 & 1476.41 & 1490.52 & 1502.30 & 1512.17 & 1519.65 & 1525.24 \\
\hline 0.05686 & 1477.90 & 1491.90 & 1503.60 & 1513.05 & 1520.50 & 1526.03 \\
\hline 0.06819 & 1478.21 & 1492.17 & 1504.24 & 1513.55 & 1520.90 & 1526.33 \\
\hline 0.08441 & 1479.56 & 1493.27 & 1504.63 & 1513.83 & 1520.97 & 1526.19 \\
\hline 0.09751 & 1480.71 & 1494.29 & 1505.51 & 1514.57 & 1521.56 & 1526.68 \\
\hline 0.11443 & 1482.25 & 1495.65 & 1506.77 & 1515.65 & 1522.52 & 1527.51 \\
\hline 0.12739 & 1482.25 & 1495.51 & 1506.44 & 1515.21 & 1521.98 & 1526.85 \\
\hline 0.13981 & 1483.20 & 1496.39 & 1507.26 & 1515.91 & 1522.56 & 1527.30 \\
\hline 0.15325 & 1485.56 & 1498.57 & 1509.24 & 1517.73 & 1524.22 & 1528.80 \\
\hline 0.16465 & 1486.58 & 1499.47 & 1510.04 & 1518.37 & 1524.73 & 1529.20 \\
\hline 0.17467 & 1487.14 & 1499.93 & 1510.38 & 1518.69 & 1524.96 & 1529.37 \\
\hline 0.19849 & 1488.69 & 1501.69 & 1511.87 & 1519.88 & 1525.93 & 1530.10 \\
\hline \multicolumn{7}{|c|}{ Water + DL-alanine $(0.438 \mathrm{~m})+\mathrm{SDS}$} \\
\hline 0.00082 & 1515.27 & 1528.87 & 1540.23 & 1549.44 & 1556.75 & 1561.98 \\
\hline 0.00156 & 1515.43 & 1529.01 & 1540.39 & 1549.69 & 1556.88 & 1562.06 \\
\hline 0.01554 & 1517.68 & 1530.96 & 1542.38 & 1551.49 & 1558.95 & 1563.20 \\
\hline 0.03025 & 1518.78 & 1531.89 & 1543.35 & 1552.11 & 1559.64 & 1563.77 \\
\hline 0.05630 & 1520.58 & 1533.47 & 1544.84 & 1553.26 & 1559.38 & 1564.65 \\
\hline 0.06954 & 1521.54 & 1534.43 & 1545.50 & 1553.78 & 1559.86 & 1564.98 \\
\hline 0.07950 & 1522.27 & 1535.10 & 1545.93 & 1554.17 & 1560.13 & 1565.14 \\
\hline 0.09943 & 1523.66 & 1536.17 & 1546.60 & 1554.78 & 1560.76 & 1565.05 \\
\hline 0.11285 & 1524.72 & 1537.06 & 1547.16 & 1555.13 & 1561.15 & 1565.39 \\
\hline 0.12791 & 1525.96 & 1538.12 & 1548.10 & 1555.84 & 1561.70 & 1565.79 \\
\hline 0.14159 & 1526.84 & 1538.86 & 1548.68 & 1556.28 & 1562.02 & 1565.97 \\
\hline 0.15603 & 1527.83 & 1539.73 & 1549.32 & 1556.83 & 1562.43 & 1566.19 \\
\hline 0.16924 & 1529.19 & 1540.92 & 1550.40 & 1557.76 & 1563.17 & 1566.80 \\
\hline 0.18305 & 1529.93 & 1541.51 & 1550.87 & 1558.00 & 1563.71 & 1566.71 \\
\hline 0.19867 & 1530.93 & 1542.34 & 1551.47 & 1558.58 & 1564.11 & 1566.99 \\
\hline
\end{tabular}




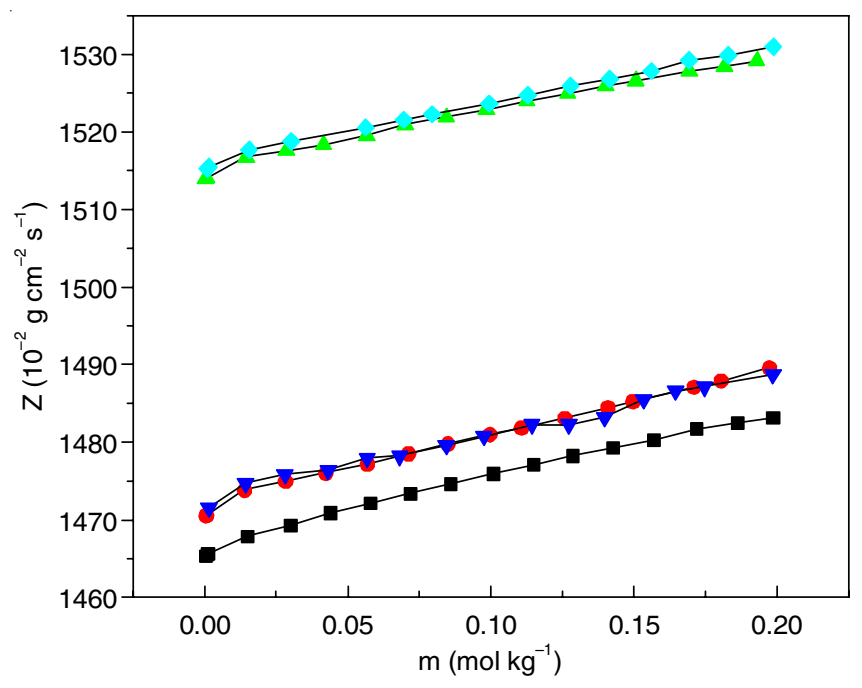

Fig. 4. Acoustic impedance (Z) of SDS in (a) water (b) $0.049 \mathrm{~m}$ glycine (c) $\Delta 0.466 \mathrm{~m}$ glycine (d) $\boldsymbol{\nabla} 0.049 \mathrm{~m}$ DL-alanine (e) $0.438 \mathrm{~m}$ DL-alanine solutions

solute-solute interaction increases for DL-alanine solvent system than glycine system. The positive acoustic impedance is an evidential parameter for solute-solvent and solute-solute interaction. The $\mathrm{Z}$ values indicate that there exists strong solutesolute along with solute-solvent interaction in aqueous and mixed aqueous solutions.

Relative association $\left(\mathbf{R}_{\mathrm{A}}\right)$ : The relative association $\left(\mathrm{R}_{\mathrm{A}}\right)$ was calculated using eqn. 4 :

$$
\mathrm{R}_{\mathrm{A}}=\left(\frac{\rho}{\rho_{\mathrm{o}}}\right)\left(\frac{\mathrm{u}_{\mathrm{o}}}{\mathrm{u}}\right)
$$

where, $\rho, \rho_{0}$ and $u, u_{0}$ are the density and ultrasonic sound velocity of solution and solvent respectively. The $R_{A}$, values of SDS in aqueous and aqueous amino acid solution at all studied temperatures are presented in Table-8 (Fig. 5). It is observed that $R_{A}$ values show slow initial decrease and then linear increase with the increase in molality of SDS. It also increases with the increase in temperatures. The $\mathrm{R}_{\mathrm{A}}$, is defined as a measure of the extent of interaction between the component molecules (solute-solute) in a real mixture relative to that in an ideal one. The positive relative association, $\mathrm{R}_{\mathrm{A}}$ demonstrates that solute-solute interactions causes association of SDS, which increases as SDS concentration.

Molar sound velocity $\left(\mathbf{R}_{\mathrm{m}}\right)$ : The molar sound velocity $\left(\mathrm{R}_{\mathrm{m}}\right)$ was calculated using eqn. 5:

TABLE-8

RELATIVE ASSOCIATION ( $\mathrm{R}_{\star}$ ) OF SDS IN AQUEOUS AND AQUEOUS AMINO ACID SOLUTIONS AT 288.15, 293.15, 298.15, 303.15, 308.15 AND 313.15 K, RESPECTIVELY

\begin{tabular}{|c|c|c|c|c|c|c|}
\hline $\mathrm{m}\left(\mathrm{mol} \mathrm{kg}^{-1}\right)$ & $298.15 \mathrm{~K}$ & $303.15 \mathrm{~K}$ & $308.15 \mathrm{~K}$ & $313.15 \mathrm{~K}$ & $318.15 \mathrm{~K}$ & $323.15 \mathrm{~K}$ \\
\hline \multicolumn{7}{|c|}{ Water + SDS } \\
\hline 0.00034 & 0.99981 & 0.99984 & 0.99995 & 0.99989 & 0.99992 & 0.99989 \\
\hline 0.00089 & 0.99970 & 0.99988 & 0.99985 & 0.99986 & 0.99979 & 0.99977 \\
\hline 0.00136 & 0.99971 & 0.99985 & 0.99985 & 0.99985 & 0.99973 & 0.99975 \\
\hline 0.01499 & 0.99966 & 1.00011 & 0.99977 & 0.99975 & 0.99993 & 0.99999 \\
\hline 0.02999 & 1.00013 & 1.00006 & 1.00030 & 1.00053 & 1.00061 & 1.00078 \\
\hline 0.04398 & 1.00035 & 1.00062 & 1.00084 & 1.00115 & 1.00138 & 1.00160 \\
\hline 0.05807 & 1.00078 & 1.00114 & 1.00142 & 1.00185 & 1.00212 & 1.00242 \\
\hline 0.07199 & 1.00122 & 1.00169 & 1.00198 & 1.00252 & 1.00287 & 1.00325 \\
\hline 0.08594 & 1.00165 & 1.00220 & 1.00256 & 1.00317 & 1.00360 & 1.00404 \\
\hline 0.10085 & 1.00211 & 1.00273 & 1.00318 & 1.00386 & 1.00436 & 1.00488 \\
\hline 0.11498 & 1.00254 & 1.00322 & 1.00373 & 1.00446 & 1.00503 & 1.00562 \\
\hline 0.12860 & 1.00292 & 1.00369 & 1.00426 & 1.00507 & 1.00572 & 1.00636 \\
\hline 0.14257 & 1.00339 & 1.00415 & 1.00479 & 1.00565 & 1.00633 & 1.00703 \\
\hline 0.15700 & 1.00397 & 1.00466 & 1.00530 & 1.00626 & 1.00700 & 1.00774 \\
\hline 0.17194 & 1.00477 & 1.00526 & 1.00602 & 1.00708 & 1.00794 & 1.00880 \\
\hline 0.18610 & 1.00517 & 1.00572 & 1.00650 & 1.00770 & 1.00862 & 1.00956 \\
\hline 0.19845 & 1.00556 & 1.00605 & 1.00681 & 1.00818 & 1.00920 & 1.01021 \\
\hline \multicolumn{7}{|c|}{ Water + Glycine $(0.049 \mathrm{~m})+$ SDS } \\
\hline 0.00035 & 0.99993 & 0.99998 & 1.00000 & 1.00001 & 0.99996 & 0.99996 \\
\hline 0.00062 & 0.99992 & 0.99990 & 0.99998 & 0.99998 & 0.99994 & 0.99994 \\
\hline 0.01394 & 0.99920 & 0.99911 & 0.99965 & 0.99972 & 0.99982 & 0.99984 \\
\hline 0.02828 & 0.99969 & 1.00005 & 1.00022 & 1.00045 & 1.00061 & 1.00069 \\
\hline 0.04222 & 1.00033 & 1.00065 & 1.00089 & 1.00115 & 1.00136 & 1.00158 \\
\hline 0.05679 & 1.00079 & 1.00114 & 1.00147 & 1.00178 & 1.00207 & 1.00236 \\
\hline 0.07115 & 1.00128 & 1.00171 & 1.00212 & 1.00246 & 1.00286 & 1.00321 \\
\hline 0.08490 & 1.00171 & 1.00220 & 1.00268 & 1.00312 & 1.00355 & 1.00398 \\
\hline 0.09953 & 1.00213 & 1.00269 & 1.00324 & 1.00380 & 1.00428 & 1.00478 \\
\hline 0.11068 & 1.00249 & 1.00311 & 1.00372 & 1.00431 & 1.00486 & 1.00543 \\
\hline 0.12577 & 1.00292 & 1.00363 & 1.00433 & 1.00499 & 1.00563 & 1.00625 \\
\hline 0.14084 & 1.00336 & 1.00413 & 1.00490 & 1.00568 & 1.00638 & 1.00710 \\
\hline 0.14951 & 1.00362 & 1.00445 & 1.00525 & 1.00611 & 1.00682 & 1.00758 \\
\hline 0.17089 & 1.00422 & 1.00516 & 1.00609 & 1.00699 & 1.00787 & 1.00873 \\
\hline 0.18048 & 1.00449 & 1.00549 & 1.00646 & 1.00743 & 1.00836 & 1.00927 \\
\hline 0.19721 & 1.00521 & 1.00606 & 1.00712 & 1.00816 & 1.00917 & 1.01015 \\
\hline
\end{tabular}




\begin{tabular}{|c|c|c|c|c|c|c|}
\hline \multicolumn{7}{|c|}{ Water + Glycine $(0.466 \mathrm{~m})+\mathrm{SDS}$} \\
\hline 0.00034 & 0.99995 & 0.99994 & 0.99994 & 0.99994 & 1.00003 & 1.00002 \\
\hline 0.00064 & 0.99991 & 0.99991 & 0.99993 & 0.99993 & 0.99998 & 0.99997 \\
\hline 0.01455 & 0.99950 & 0.99964 & 0.99977 & 0.99988 & 1.00000 & 0.99976 \\
\hline 0.02873 & 1.00003 & 1.00022 & 1.00043 & 1.00060 & 1.00095 & 1.00072 \\
\hline 0.04149 & 1.00047 & 1.00072 & 1.00099 & 1.00123 & 1.00146 & 1.00130 \\
\hline 0.05671 & 1.00096 & 1.00130 & 1.00164 & 1.00194 & 1.00226 & 1.00222 \\
\hline 0.07014 & 1.00141 & 1.00182 & 1.00224 & 1.00264 & 1.00301 & 1.00322 \\
\hline 0.08458 & 1.00186 & 1.00233 & 1.00282 & 1.00328 & 1.00374 & 1.00385 \\
\hline 0.09834 & 1.00227 & 1.00282 & 1.00338 & 1.00393 & 1.00447 & 1.00471 \\
\hline 0.11258 & 1.00272 & 1.00335 & 1.00400 & 1.00462 & 1.00522 & 1.00534 \\
\hline 0.12687 & 1.00314 & 1.00385 & 1.00457 & 1.00527 & 1.00595 & 1.00635 \\
\hline 0.14028 & 1.00357 & 1.00434 & 1.00513 & 1.00589 & 1.00664 & 1.00717 \\
\hline 0.15060 & 1.00387 & 1.00469 & 1.00554 & 1.00636 & 1.00717 & 1.00792 \\
\hline 0.16942 & 1.00442 & 1.00535 & 1.00630 & 1.00721 & 1.00811 & 1.00899 \\
\hline 0.18166 & 1.00480 & 1.00579 & 1.00680 & 1.00777 & 1.00873 & 1.00965 \\
\hline 0.19292 & 1.00515 & 1.00621 & 1.00727 & 1.00830 & 1.00937 & 1.01032 \\
\hline \multicolumn{7}{|c|}{ Water + DL-alanine $(0.049 \mathrm{~m})+\mathrm{SDS}$} \\
\hline 0.00150 & 0.99973 & 0.99978 & 0.99976 & 0.99975 & 0.99973 & 0.99987 \\
\hline 0.01442 & 0.99882 & 0.99946 & 0.99954 & 0.99963 & 0.99935 & 0.99961 \\
\hline 0.02820 & 0.99933 & 1.00004 & 1.00015 & 1.00032 & 1.00038 & 1.00039 \\
\hline 0.04313 & 1.00029 & 1.00064 & 1.00087 & 1.00091 & 1.00123 & 1.00151 \\
\hline 0.05686 & 1.00060 & 1.00101 & 1.00128 & 1.00159 & 1.00189 & 1.00219 \\
\hline 0.06819 & 1.00125 & 1.00165 & 1.00164 & 1.00203 & 1.00239 & 1.00274 \\
\hline 0.08441 & 1.00178 & 1.00232 & 1.00275 & 1.00318 & 1.00363 & 1.00409 \\
\hline 0.09751 & 1.00218 & 1.00279 & 1.00329 & 1.00378 & 1.00431 & 1.00482 \\
\hline 0.11443 & 1.00246 & 1.00315 & 1.00371 & 1.00429 & 1.00488 & 1.00546 \\
\hline 0.12739 & 1.00303 & 1.00375 & 1.00441 & 1.00505 & 1.00568 & 1.00631 \\
\hline 0.13981 & 1.00347 & 1.00427 & 1.00494 & 1.00563 & 1.00633 & 1.00703 \\
\hline 0.15325 & 1.00363 & 1.00453 & 1.00530 & 1.00608 & 1.00687 & 1.00766 \\
\hline 0.16465 & 1.00403 & 1.00498 & 1.00581 & 1.00667 & 1.00753 & 1.00836 \\
\hline 0.17467 & 1.00422 & 1.00522 & 1.00612 & 1.00698 & 1.00788 & 1.00876 \\
\hline 0.19849 & 1.00522 & 1.00604 & 1.00708 & 1.00810 & 1.00911 & 1.01010 \\
\hline \multicolumn{7}{|c|}{ Water + DL-alanine $(0.438 \mathrm{~m})+\mathrm{SDS}$} \\
\hline 0.00082 & 1.00003 & 1.00003 & 1.00002 & 1.00001 & 0.99995 & 1.00002 \\
\hline 0.00156 & 0.99995 & 0.99997 & 0.99994 & 0.99987 & 0.99988 & 0.99999 \\
\hline 0.01554 & 0.99983 & 1.00003 & 0.99995 & 0.99999 & 0.99980 & 1.00047 \\
\hline 0.03025 & 1.00042 & 1.00068 & 1.00055 & 1.00076 & 1.00052 & 1.00127 \\
\hline 0.05630 & 1.00139 & 1.00175 & 1.00162 & 1.00204 & 1.00265 & 1.00260 \\
\hline 0.06954 & 1.00189 & 1.00221 & 1.00226 & 1.00274 & 1.00335 & 1.00339 \\
\hline 0.07950 & 1.00224 & 1.00259 & 1.00276 & 1.00324 & 1.00391 & 1.00402 \\
\hline 0.09943 & 1.00299 & 1.00348 & 1.00389 & 1.00433 & 1.00496 & 1.00552 \\
\hline 0.11285 & 1.00335 & 1.00393 & 1.00452 & 1.00508 & 1.00565 & 1.00622 \\
\hline 0.12791 & 1.00373 & 1.00439 & 1.00504 & 1.00570 & 1.00635 & 1.00700 \\
\hline 0.14159 & 1.00417 & 1.00489 & 1.00563 & 1.00635 & 1.00707 & 1.00780 \\
\hline 0.15603 & 1.00459 & 1.00541 & 1.00624 & 1.00704 & 1.00784 & 1.00863 \\
\hline 0.16924 & 1.00466 & 1.00556 & 1.00647 & 1.00739 & 1.00828 & 1.00914 \\
\hline 0.18305 & 1.00519 & 1.00616 & 1.00712 & 1.00815 & 1.00882 & 1.01009 \\
\hline 0.19867 & 1.00568 & 1.00674 & 1.00782 & 1.00885 & 1.00962 & 1.01092 \\
\hline
\end{tabular}

$$
\mathrm{R}_{\mathrm{m}}=\mathrm{Vu}^{1 / 3}
$$

where, $\mathrm{V}$ and $\mathrm{u}$ are the molar volume and ultrasonic sound velocity respectively. The $\mathrm{R}_{\mathrm{m}}$, values of SDS in aqueous and aqueous amino acid solution at all studied temperatures are presented in Table-9 and graphically represented in Fig. 6. The $R_{m}$ values show an asymptotic increase with the increase in molality of SDS. The increase $\mathrm{R}_{\mathrm{m}}$ values with the molality of SDS are an evidence of the structure of solution due to solute-solute, solute-cosolvent and solute-solvent interactions. The asymptotic nature reveals that the reinforcement of structure of solution approaches to attain a maximum value.

\section{Conclusion}

Adiabatic compressibility $\left(\beta_{\mathrm{s}}\right)$, apparent molar adiabatic compressibility $\left(\phi_{\mathrm{K}}\right)$, critical micelle concentration $(\mathrm{CMC})$, acoustic impedance $(\mathrm{Z})$, relative association $\left(\mathrm{R}_{\mathrm{A}}\right)$ and molar sound velocity $\left(\mathrm{R}_{\mathrm{m}}\right)$ of SDS in aqueous and aqueous amino acid solutions are calculated by the measurement of density and ultrasonic velocity at six different temperatures and atmospheric pressure. The $\phi_{\mathrm{K}}$ values increases due to the dehydration of hydration sphere of SDS by the interactions of amino acids. Critical micelle concentration values of SDS in aqueous amino acid solution are larger than aqueous solution indicate that 


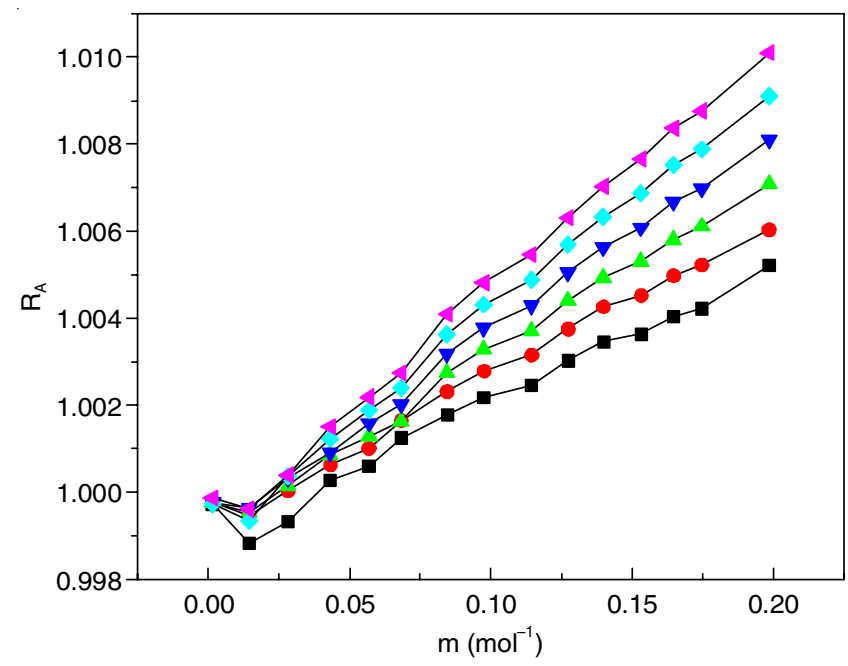

Fig. 5. Relative association $\left(\mathrm{R}_{\mathrm{A}}\right)$ of SDS in $0.049 \mathrm{~m}$ DL-alanine as a function of molality at different temperatures; $288.15 \mathrm{~K}$, $293.15 \mathrm{~K}, \boldsymbol{\Delta}$ $298.15 \mathrm{~K}, \boldsymbol{\nabla} 303.15 \mathrm{~K}, \diamond 308.15 \mathrm{~K}, \triangleleft 313.15 \mathrm{~K}$

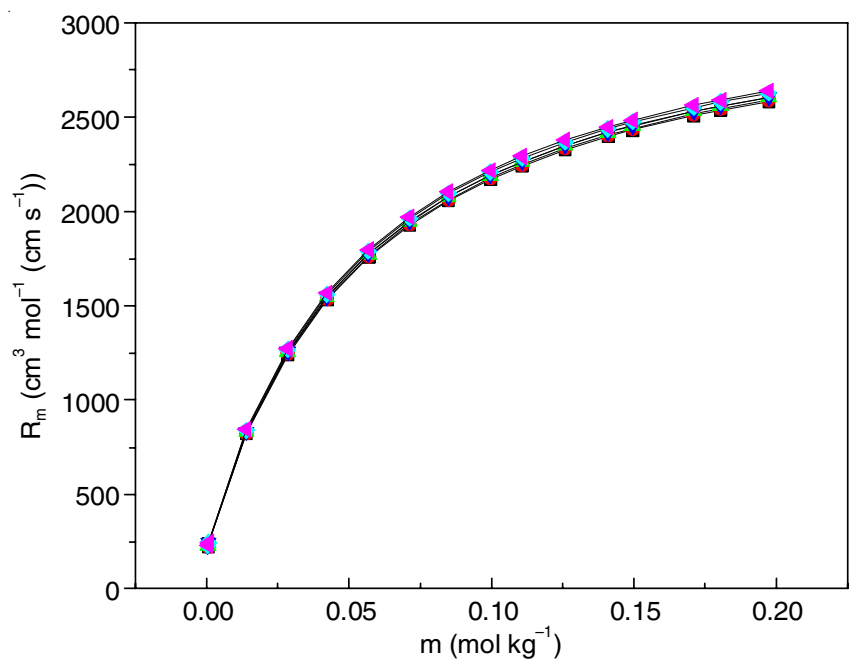

Fig. 6. Molar sound velocity $\left(\mathrm{R}_{\mathrm{m}}\right)$ of $\operatorname{SDS}$ in $0.049 \mathrm{~m}$ glycine as a function of molality at different temperatures; $-288.15 \mathrm{~K}, \bullet 293.15 \mathrm{~K}, \boldsymbol{\Delta}$ $298.15 \mathrm{~K}, \boldsymbol{\nabla} 303.15 \mathrm{~K}, \diamond 308.15 \mathrm{~K}, \triangleleft 313.15 \mathrm{~K}$

TABLE-9

MOLAR SOUND VELOCITY $\left(\mathrm{R}_{\mathrm{m}}, \mathrm{cm}^{3} \mathrm{~mol}^{-1}\left(\mathrm{~cm} \mathrm{~s}^{-1}\right)^{1 / 3}\right)$ OF SDS IN AQUEOUS AND AQUEOUS AMINO ACID SOLUTIONS AT 288.15, 293.15, 298.15, 303.15, 308.15 AND 313.15 K, RESPECTIVELY

\begin{tabular}{|c|c|c|c|c|c|c|}
\hline $\mathrm{m}\left(\mathrm{mol} \mathrm{kg}{ }^{-1}\right)$ & $298.15 \mathrm{~K}$ & $303.15 \mathrm{~K}$ & $308.15 \mathrm{~K}$ & $313.15 \mathrm{~K}$ & $318.15 \mathrm{~K}$ & $323.15 \mathrm{~K}$ \\
\hline \multicolumn{7}{|c|}{ Water + SDS } \\
\hline 0.00034 & 223.78 & 224.81 & 226.25 & 226.71 & 228.77 & 230.06 \\
\hline 0.00089 & 253.60 & 254.76 & 256.39 & 256.92 & 259.25 & 260.71 \\
\hline 0.00136 & 278.63 & 279.90 & 281.69 & 282.27 & 284.82 & 286.42 \\
\hline 0.01499 & 858.93 & 862.64 & 867.97 & 869.43 & 877.07 & 881.66 \\
\hline 0.02999 & 1282.77 & 1288.12 & 1295.88 & 1297.73 & 1308.89 & 1315.38 \\
\hline 0.04398 & 1562.27 & 1568.61 & 1577.91 & 1579.86 & 1593.25 & 1600.87 \\
\hline 0.05807 & 1773.66 & 1780.72 & 1791.11 & 1793.05 & 1808.13 & 1816.52 \\
\hline 0.07199 & 1936.26 & 1943.79 & 1955.07 & 1956.89 & 1973.23 & 1982.15 \\
\hline 0.08594 & 2066.66 & 2074.55 & 2086.48 & 2088.18 & 2105.54 & 2114.86 \\
\hline 0.10085 & 2179.97 & 2188.16 & 2200.67 & 2202.18 & 2220.43 & 2230.08 \\
\hline 0.11498 & 2268.82 & 2277.23 & 2290.17 & 2291.53 & 2310.48 & 2320.35 \\
\hline 0.12860 & 2341.29 & 2349.83 & 2363.12 & 2364.33 & 2383.83 & 2393.89 \\
\hline 0.14257 & 2405.00 & 2413.73 & 2427.31 & 2428.37 & 2448.41 & 2458.62 \\
\hline 0.15700 & 2461.79 & 2470.60 & 2484.47 & 2485.35 & 2505.88 & 2516.22 \\
\hline 0.17194 & 2512.25 & 2521.05 & 2535.45 & 2535.77 & 2557.02 & 2567.42 \\
\hline 0.18610 & 2554.82 & 2563.67 & 2578.10 & 2578.40 & 2599.83 & 2610.27 \\
\hline 0.19845 & 2587.96 & 2596.87 & 2611.28 & 2611.56 & 2633.11 & 2643.59 \\
\hline \multicolumn{7}{|c|}{ Water + Glycine $(0.049 \mathrm{~m})+\mathrm{SDS}$} \\
\hline 0.00035 & 224.10 & 225.12 & 226.57 & 227.03 & 229.10 & 230.39 \\
\hline 0.00062 & 239.12 & 240.21 & 241.75 & 242.24 & 244.46 & 245.83 \\
\hline 0.01394 & 822.28 & 825.83 & 830.95 & 832.34 & 839.71 & 844.12 \\
\hline 0.02828 & 1241.90 & 1247.06 & 1254.62 & 1256.38 & 1267.26 & 1273.58 \\
\hline 0.04222 & 1531.04 & 1537.23 & 1546.36 & 1548.25 & 1561.48 & 1568.96 \\
\hline 0.05679 & 1756.01 & 1762.95 & 1773.28 & 1775.16 & 1790.16 & 1798.47 \\
\hline 0.07115 & 1926.59 & 1934.03 & 1945.24 & 1947.06 & 1963.37 & 1972.26 \\
\hline 0.08490 & 2056.77 & 2064.58 & 2076.44 & 2078.12 & 2095.47 & 2104.74 \\
\hline 0.09953 & 2169.73 & 2177.83 & 2190.27 & 2191.74 & 2209.99 & 2219.59 \\
\hline 0.11068 & 2242.20 & 2250.47 & 2263.26 & 2264.61 & 2283.44 & 2293.22 \\
\hline 0.12577 & 2325.82 & 2334.26 & 2347.41 & 2348.62 & 2368.09 & 2378.09 \\
\hline 0.14084 & 2396.12 & 2404.69 & 2418.22 & 2419.16 & 2439.21 & 2449.35 \\
\hline 0.14951 & 2431.76 & 2440.38 & 2454.09 & 2454.88 & 2475.25 & 2485.45 \\
\hline 0.17089 & 2507.71 & 2516.43 & 2530.48 & 2531.03 & 2552.00 & 2562.34 \\
\hline 0.18048 & 2537.15 & 2545.89 & 2560.08 & 2560.50 & 2581.70 & 2592.09 \\
\hline 0.19721 & 2582.60 & 2591.37 & 2605.77 & 2605.96 & 2627.57 & 2638.02 \\
\hline
\end{tabular}




\begin{tabular}{|c|c|c|c|c|c|c|}
\hline \multicolumn{7}{|c|}{ Water + Glycine $(0.466 \mathrm{~m})+\mathrm{SDS}$} \\
\hline 0.00034 & 223.88 & 224.89 & 226.38 & 226.76 & 228.91 & 230.20 \\
\hline 0.00064 & 240.57 & 241.65 & 243.25 & 243.66 & 245.97 & 247.34 \\
\hline 0.01455 & 842.81 & 846.35 & 851.75 & 852.84 & 860.67 & 865.24 \\
\hline 0.02873 & 1250.28 & 1255.32 & 1263.10 & 1264.40 & 1275.68 & 1282.13 \\
\hline 0.04149 & 1513.88 & 1519.80 & 1529.05 & 1530.34 & 1543.90 & 1551.42 \\
\hline 0.05671 & 1748.91 & 1755.54 & 1766.06 & 1767.24 & 1782.70 & 1791.07 \\
\hline 0.07014 & 1908.22 & 1915.30 & 1926.64 & 1927.64 & 1944.43 & 1953.19 \\
\hline 0.08458 & 2045.21 & 2052.64 & 2064.68 & 2065.50 & 2083.34 & 2092.66 \\
\hline 0.09834 & 2151.43 & 2159.09 & 2171.65 & 2172.26 & 2190.94 & 2200.52 \\
\hline 0.11258 & 2242.65 & 2250.50 & 2263.49 & 2263.90 & 2283.31 & 2293.26 \\
\hline 0.12687 & 2319.64 & 2327.62 & 2340.99 & 2341.17 & 2361.20 & 2371.19 \\
\hline 0.14028 & 2381.31 & 2389.39 & 2403.03 & 2403.03 & 2423.56 & 2433.62 \\
\hline 0.15060 & 2423.28 & 2431.41 & 2445.25 & 2445.09 & 2465.96 & 2475.95 \\
\hline 0.16942 & 2489.53 & 2497.73 & 2511.87 & 2511.43 & 2532.85 & 2542.92 \\
\hline 0.18166 & 2526.88 & 2535.11 & 2549.41 & 2548.81 & 2570.53 & 2580.67 \\
\hline 0.19292 & 2557.81 & 2566.03 & 2580.48 & 2579.72 & 2601.60 & 2611.86 \\
\hline \multicolumn{7}{|c|}{ Water + DL-alanine $(0.049 \mathrm{~m})+\mathrm{SDS}$} \\
\hline 0.00150 & 286.22 & 287.51 & 289.35 & 289.92 & 292.56 & 294.19 \\
\hline 0.01442 & 839.89 & 843.48 & 848.69 & 850.09 & 857.59 & 862.07 \\
\hline 0.02820 & 1241.01 & 1246.14 & 1253.68 & 1255.43 & 1266.26 & 1272.55 \\
\hline 0.04313 & 1548.37 & 1554.58 & 1563.78 & 1565.75 & 1579.03 & 1586.54 \\
\hline 0.05686 & 1758.29 & 1765.20 & 1775.51 & 1777.37 & 1792.34 & 1800.64 \\
\hline 0.06819 & 1896.07 & 1903.44 & 1914.69 & 1916.45 & 1932.49 & 1941.26 \\
\hline 0.08441 & 2054.08 & 2061.85 & 2073.67 & 2075.31 & 2092.60 & 2101.83 \\
\hline 0.09751 & 2156.85 & 2164.88 & 2177.21 & 2178.71 & 2196.78 & 2206.32 \\
\hline 0.11443 & 2266.08 & 2274.37 & 2287.24 & 2288.54 & 2307.47 & 2317.30 \\
\hline 0.12739 & 2335.87 & 2344.31 & 2357.53 & 2358.64 & 2378.17 & 2388.18 \\
\hline 0.13981 & 2393.58 & 2402.14 & 2415.63 & 2416.63 & 2436.58 & 2446.70 \\
\hline 0.15325 & 2447.89 & 2456.51 & 2470.24 & 2471.02 & 2491.42 & 2501.64 \\
\hline 0.16465 & 2488.64 & 2497.31 & 2511.25 & 2511.85 & 2532.58 & 2542.88 \\
\hline 0.17467 & 2521.44 & 2530.15 & 2544.22 & 2544.74 & 2565.73 & 2576.09 \\
\hline 0.19849 & 2587.76 & 2596.78 & 2611.12 & 2611.30 & 2632.88 & 2643.31 \\
\hline \multicolumn{7}{|c|}{ Water + DL-alanine $(0.438 \mathrm{~m})+\mathrm{SDS}$} \\
\hline 0.00082 & 253.23 & 254.33 & 255.96 & 256.39 & 258.75 & 260.17 \\
\hline 0.00156 & 292.73 & 293.99 & 295.88 & 296.36 & 299.09 & 300.71 \\
\hline 0.01554 & 885.58 & 889.24 & 894.84 & 895.96 & 903.94 & 908.33 \\
\hline 0.03025 & 1297.41 & 1302.47 & 1310.28 & 1311.59 & 1323.05 & 1329.16 \\
\hline 0.05630 & 1756.38 & 1762.96 & 1773.35 & 1774.49 & 1789.61 & 1797.64 \\
\hline 0.06954 & 1915.48 & 1922.40 & 1933.45 & 1934.46 & 1950.83 & 1959.37 \\
\hline 0.07950 & 2014.39 & 2021.56 & 2033.08 & 2033.99 & 2051.13 & 2059.94 \\
\hline 0.09943 & 2173.05 & 2180.75 & 2193.08 & 2193.67 & 2212.09 & 2221.35 \\
\hline 0.11285 & 2258.31 & 2266.02 & 2278.72 & 2279.17 & 2298.21 & 2307.67 \\
\hline 0.12791 & 2338.60 & 2346.44 & 2359.54 & 2359.73 & 2379.40 & 2389.03 \\
\hline 0.14159 & 2400.74 & 2408.68 & 2422.05 & 2422.04 & 2442.22 & 2451.96 \\
\hline 0.15603 & 2457.25 & 2465.25 & 2478.84 & 2478.63 & 2499.27 & 2509.10 \\
\hline 0.16924 & 2502.25 & 2510.27 & 2524.09 & 2523.64 & 2544.64 & 2554.54 \\
\hline 0.18305 & 2543.86 & 2551.91 & 2565.93 & 2565.24 & 2586.63 & 2596.51 \\
\hline 0.19867 & 2585.36 & 2593.41 & 2607.56 & 2606.70 & 2628.41 & 2638.41 \\
\hline
\end{tabular}

glycine and alanine are water structure breakers. The $\mathrm{Z}$ values indicate strong solute-solute along with solute-solvent interaction in aqueous and aqueous amino acid solutions. The $\mathrm{R}_{\mathrm{A}}$ values identify the interaction between the solute-solute molecules in a real mixture. The $\mathrm{R}_{\mathrm{m}}$ values give an evidence for strengthening the structure of solution due to solute-cosolutesolvent interactions. So, aforesaid information's are valuable for understanding surfactant-protein interactions and for other application of surfactant in pharmaceuticals.

\section{ACKNOWLEDGEMENTS}

The authors are thankful to the Department of Chemistry, University of Rajshahi, Bangladesh for providing financial support and laboratory facilities to carry out this research work.

\section{CONFLICT OF INTEREST}

The authors declare that there is no conflict of interests regarding the publication of this article.

\section{REFERENCES}

1. T.F. Tadros, Applied Surfactants, Wiley-VCH: Weinheim (2005)

2. O. Kirk, T.V. Borchert and C.C. Fuglsang, Curr. Opin. Biotechnol., 13, 345 (2002);

https://doi.org/10.1016/S0958-1669(02)00328-2.

3. M. Lee and J.S. Dordick, Curr. Opin. Biotechnol., 13, 376 (2002); https://doi.org/10.1016/S0958-1669(02)00337-3.

4. A.L. Shapiro, E. Viñuela and J. V. Maizel Jr., Biochem. Biophys. Res. Commun., 28, 815 (1967);

https://doi.org/10.1016/0006-291X(67)90391-9.

5. K. Weber and M. Osborn, J. Biol. Chem., 244, 4406 (1969). 
6. K. Duquesne and J.N. Sturgis, Methods Mol. Biol., 601, 205 (2010); https://doi.org/10.1007/978-1-60761-344-2 13.

7. G.G. Prive, Methods, 41, 388 (2007); https://doi.org/10.1016/j.ymeth.2007.01.007.

8. G. Garavito and S.J. Ferguson-Miller, Biol. Chem., 276, 32403 (2001); https://doi.org/10.1074/jbc.R100031200.

9. A. Ali, M. Tariq, R. Patel and F.A. Ittoo, Colloid Polym. Sci., 286, 183 (2008); https://doi.org/10.1007/s00396-007-1750-5.

10. A. Ali and N.H. Ansari, J. Surfactants Deterg., 13, 441 (2010); https://doi.org/10.1007/s11743-010-1221-8.

11. S.K. Singh, A. Kundu and N. Kishore, J. Chem. Thermodyn., 36, 7 (2004); https://doi.org/10.1016/j.jct.2003.09.010.

12. M. Vasilescu, D. Angelescu, M. Almgren and A. Valstar, Langmuir, 15, 2635 (1999); https://doi.org/10.1021/la981424y.

13. Y. Moriyama and K. Takeda, Langmuir, 15, 2003 (1999); https://doi.org/10.1021/la981442f

14. T. Cserháti, E. Forgács, Z. Deyl, I. Miksik and A. Eckhardt, J. Chromatogr. A, 910, 137 (2001); https://doi.org/10.1016/S0021-9673(00)01191-2.

15. R.B. Singh, S. Mahanta and N. Guchhait, Chem. Phys. Lett., 463, 183 (2008); https://doi.org/10.1016/j.cplett.2008.08.017.

16. Y. Moriyama, E. Watanabe, K. Kobayashi, H. Harano, E. Inui and K. Takeda, J. Phys. Chem. B, 112, 16585 (2008); https://doi.org/10.1021/jp8067624.

17. M.N. Jones, H.A. Skinner, E. Tipping and A. Wilkinson, Biochem. J., 135, 231 (1973); https://doi.org/10.1042/bj1350231.
18. C. Blinkhorn and M.N. Jones, Biochem. J., 135, 547 (1973); https://doi.org/10.1042/bj1350547.

19. C. Si-Qing, F. Xian-Gang, Y. Jian-Fang and L. Jie-Hua, J. Dispers. Sci. Technol., 28, 297 (2007); https://doi.org/10.1080/01932690601062127.

20. A.A. Moosavi-Movahedi, M. Gharanfoli, K. Nazari, M. Shamsipur, J. Chamani, B. Hemmateenejad, M. Alavi, A. Shokrollahi, M. HabibiRezaei, C. Sorenson and N. Sheibani, Colloids Surf. B Biointerfaces, 43, 150 (2005); https://doi.org/10.1016/j.colsurfb.2005.04.008.

21. P. Sharma, S. Chauhan, M.S. Chauhan and V.K. Syal, Indian J. Pure Appl. Phys., 46, 839 (2008).

22. P. Science and S. De Compostela, Colloid Polym. Sci., 114, 108 (1994).

23. S.E. Burke, S.L. Andrecyk and R. Palepu, Colloid Polym. Sci., 279, 131 (2001); https://doi.org/10.1007/s003960000402.

24. S. Chauhan, D.S. Rana, K. Rana, M.S. Chauhan and A. Umar, J. Comput. Theor. Nanosci., 5, 178 (2012).

25. P.C. Pal and S.P. Das, Int. J. Pharm., 4, 45 (2015).

26. H. Kumar and K. Kaur, J. Chem. Eng. Data, 58, 203 (2013); https://doi.org/10.1021/je3006425.

27. M.R. Khatun, M.M. Islam, F.R. Rima and M.N. Islam, J. Chem. Eng. Data, 61, 102 (2016); https://doi.org/10.1021/acs.jced.5b00317.

28. R. Khatun, R. Sultana and R.K. Nath, Orient. J. Chem., 34, 1755 (2018); https://doi.org/10.13005/ojc/340407.

29. R. Khatun and N. Islam, Orient. J. Chem., 28, 165 (2012); https://doi.org/10.13005/ojc/280123.

30. D. Shaw, Introduction to Colloid \& Surface Chemistry, edn 4 (2013). 